\title{
Störung und >Entstörung \ in Christian Krachts und Eckhart Nickels Gebrauchsanweisung für Kathmandu und Nepal (2009/2012)
}

\section{Zu einer Poetik des »Knowing without going «}

\author{
Matthias N. Lorenz $\cdot$ Christine Riniker
}

Online publiziert: 5. Juli 2018

(C) Springer-Verlag GmbH Deutschland, ein Teil von Springer Nature 2018

Zusammenfassung Der Beitrag befasst sich mit unterschiedlichen Aspekten von Ordnung, Störung und partieller >Entstörung « in Christian Krachts und Eckhart Nickels Gebrauchsanweisung für Kathmandu und Nepal (2009) sowie in deren überarbeiteter Neuausgabe (2012). Es wird analysiert, wie sich der Band innerhalb der Reihe des Piper-Verlags positioniert, welche reiseliterarischen Traditionslinien aufgerufen und wie die auf einen literarischen Reiseführer eingestellten Rezeptionserwartungen durch den Text enttäuscht werden. Dabei wird ein durchtriebenes Spiel deutlich, das Fragen politischer Korrektheit und reiseliterarischer Redlichkeit aufwirft. Ausgehend von den Vorwürfen gegen die Popliteraten der 1990er Jahre, sie seien kolonialapologetische Dandys, wird hier eine Lesart in Stellung gebracht, die im intertextuellen und unzuverlässigen Spiel ein ebenso subtiles wie kritisches politisches Statement entdeckt.

Schlüsselwörter Christian Kracht · Eckhart Nickel · Störung · Reiseführer · Reiseliteratur · Popliteratur · Dandyismus · Ironie · Orientalismus · Political Correctness

\footnotetext{
M. N. Lorenz $(\bowtie) \cdot$ C. Riniker

Institut für Germanistik, Universität Bern, Bern, Schweiz

E-Mail: matthias.lorenz@germ.unibe.ch

C. Riniker

E-Mail: christine.riniker@germ.unibe.ch

M. N. Lorenz

Department of Modern Foreign Languages, Stellenbosch University, Stellenbosch, Südafrika
} 


\title{
Disruption and Restitution of Order in Christian Kracht's und Eckhart Nickel's Gebrauchsanweisung für Kathmandu und Nepal (2009/2012)
}

The Poetics of »Knowing Without Going«

\begin{abstract}
This paper investigates different aspects of order, disruption and partial restoration in Christian Kracht's und Eckhart Nickel's Gebrauchsanweisung für Kathmandu und Nepal (2009) and the revised edition (2012). The analysis focuses on the positioning of the text within the book series of Piper publishing as well as on the traditions of the genre of travel writing on which the authors allude to. Furthermore we question, to what extent the readers expectations are being disappointed. This in itself reveals to be an artful game that raises further questions concerning the matter of political correctness in this specific type of travel writing. In regard to the criticism of German Pop Literature and its authors - who were broadly perceived as apolitical dandies trivializing colonialism in their travelogues - , this paper presents an analysis of intertextuality and unreliability and interprets these techniques as a subtle but nevertheless critical statement.
\end{abstract}

Keywords Christian Kracht · Eckhart Nickel · Disruption · Travel Guide · Travel Literature · Pop · Dandyism · Irony · Orientalism · Political Correctness

\section{Wo gehen wir denn hin? Immer nach Hause. (Novalis)}

2009 erschien im Münchner Piper-Verlag in der eingeführten Reihe der »Gebrauchsanweisungen für ...« die Gebrauchsanweisung für Kathmandu und Nepal. Die Autoren sind Christian Kracht und Eckhart Nickel, zwei 1966 geborene Vertreter der sogenannten zweiten Generation der deutschen Popliteratur. Die PiperReihe will Reisebücher vorstellen, in denen die Autoren, so die Verlagswerbung, »ihre Eindrücke und ortskundige Geschichten aufschreiben und sich mit persönlichem Blick den Ländern, Regionen oder Städten auf ungewöhnliche und literarische Weise annähern «. ${ }^{1}$ Pilotband war 1978 die humorvolle Gebrauchsanweisung des Psychotherapeuten Paul Watzlawick für seine Wahlheimat Amerika. Der große Erfolg der Reihe - deren Gesamtauflage bei über 2,2 Millionen Exemplaren liegen soll $^{2}$ - lässt allerdings vermuten, dass die Titel oftmals analog zu Reiseführern vom Schlage eines Baedeker oder Marco Polo gekauft werden. ${ }^{3}$

Das Besondere an den Piper-Bänden ist jedoch der dezidiert subjektive Zugang der jeweiligen Autoren, unter denen sich mitunter auch einige jüngere Literaten finden. So hat Antje Rávic Strubel etwa eine Gebrauchsanweisung fürs Skifahren vorgelegt, Jakob Hein eine für Berlin, Elke Naters ist Mitautorin derjenigen für

\footnotetext{
1 Website des Piper-Verlags. In: https://www.piper.de/buecher/abenteuer-reiseberichte/gebrauchsanwei sung (2.11.2017).

2 Vgl. Website des Piper-Verlags. In: https://www.piper.de/buecher/abenteuer-reiseberichte/gebrauchsan weisung (2.11.2017).

3 Amazon.de zufolge ist etwa Kai Strittmatters Gebrauchsanweisung für China der »Bestseller Nr. 1《 in der Kategorie »Reiseführer China \& Tibet«. Vgl. Website von amazon.de. In: http://www.amazon.de/ Gebrauchsanweisung-für-China-Kai-Strittmatter/dp/3492275745/ref=sr_1_1 ?ie=UTF8\&qid=1512493460 $\&$ sr=8-1\&keywords=gebrauchsanweisung+für+china $(5.12 .2017)$.
} 
Kapstadt und Südafrika. Die allermeisten Bände widmen sich bestimmten Städten, Ländern, Gebirgen und Küsten; Titel wie die Gebrauchsanweisung für Weihnachten oder die deutsche Bahn sind jüngeren Datums und im touristischen Programm eher die Ausnahme.

Der Verlag betont in seiner Eigendarstellung, »namhafte Autoren « hierfür gewonnen zu haben und nennt als Beispiele »Kai Strittmatter, Antje Rávic Strubel, Ilija Trojanow oder Jakob Hein «. ${ }^{4}$ Auffälligerweise fehlen Christian Kracht und Eckhart Nickel in dieser kurzen Liste, die offenkundig das Schriftstellerische betonen möchte, obwohl die allermeisten Autoren der Gebrauchsanweisungen keine Literaten sind. Dies ist besonders auffällig, da mit Christian Kracht ausgerechnet der mit Abstand renommierteste Autor dieses Sets ausgeblendet wird. Hier deutet sich bereits ein gestörtes Verhältnis an, das sich möglicherweise auch darin äußert, dass der Verlag uns auf unsere Anfragen bislang noch keine Antworten geben konnte.

Im folgenden Beitrag wird gezeigt, wie der Band von Kracht und Nickel in der Piper-Reihe zu verorten ist und wie seine Platzierung in derselben auf die anderen Gebrauchsanweisungen zurückwirkt. Die Untersuchung bringt ein durchgehendes literarisches Verweissystem des Textes über Kathmandu und Nepal zutage, das eben diese maximal basale Mitteilung, die der Titel überhaupt nur zu machen scheint, ein reiseliterarisches Buch über Kathmandu und Nepal zu sein, in Zweifel zieht. Im Nachvollzug dieser Verweise wird eine komplexe Spielanordnung von Störung und Entstörung sichtbar, die zum einen gattungspoetologisch erfüllt, was Störungen zu leisten vermögen, nämlich im Regelverstoß die Regeln von Kommunikation sichtbar zu machen. ${ }^{5}$ Zum anderen wirft die Störung die Frage nach rezeptionsästhetischen und - im Kontext einer europäischen Literatur über nichteuropäische Länder, Kulturen und Menschen - nicht zuletzt auch politischen Implikationen einer insbesondere für Christian Kracht typischen Poetik auf, dessen Werke schon häufiger im Kreuzfeuer einer ethisch-moralisch motivierten Literaturkritik standen. Die Debatte um den Roman Imperium ${ }^{6}$ war nicht die erste, wohl aber die breitenwirksamste dieser Art. Gerade weil das Feuilleton nach fast zwei Jahrzehnten immer wieder heftiger Ablehnung der Werke Krachts mittlerweile mehrheitlich zu einer anderen Beurteilung gekommen ist, ohne dass sich an den Verfahren Krachts Grundlegendes geändert zu haben scheint, ${ }^{7}$ soll exemplarisch aufgezeigt werden, wie dieses Verfahren überhaupt funktioniert. Abschließend ist zu diskutieren, welche emanzipatorisch-kritischen oder aber tradierte Stereotype affirmierenden Potentiale diese Literatur hat.

\footnotetext{
4 Website des Piper-Verlags. In: https://www.piper.de/buecher/abenteuer-reiseberichte/gebrauchsanweisung (2.11.2017).

5 Vgl. Gansel, Carsten: »Einleitung«. In: Ders./Norman Ächtler (Hg.): Das »Prinzip Störung in den Geistes- und Sozialwissenschaften. Berlin (2013, S. 7-13).

6 Vgl. Winkels, Hubert (Hg.): Christian Kracht trifft Wilhelm Raabe. Die Diskussion um »Imperium « und der Wilhelm Raabe-Literaturpreis 2012. Berlin (2013).

7 Vgl. zu den fortgesetzten Mühen der etablierten Literaturkritik mit Krachts Poetik wie auch zur Kontinuität seiner Schreibverfahren Lorenz, Matthias N.: »Der freundliche Kannibale. Über den Provokationsgehalt der Figur >Christian Kracht««. In: Merkur. Deutsche Zeitschrift für europäisches Denken 68 (2014), H. 11, S. 1022-1026.
} 
Sieht man sich einige der Gebrauchsanweisungen an, so zeigen sich verschiedene Grade der Subjektivität. Alle Autorinnen und Autoren beschreiben ihr Portugal, ihr Tibet, ihr Indien. Die Schilderungen können die Form von nützlichen Insidertipps annehmen, aber ebenso persönliche Erlebnisse aufgreifen, die entweder typische interkulturelle Situationen einfangen oder etwas über die Mentalitäten der Bewohner der bereisten Regionen aussagen sollen. Die Bücher sind auch offen für Selbstdarstellungen der Autorinnen und Autoren, die sich etwa eines bestimmten Stils befleißigen, im Gegensatz zum klassischen Reiseführer auch Ironie walten lassen und sehr subjektive Bewertungen abgeben dürfen.

Krachts Co-Autor Eckhart Nickel, der bereits 2001 eine Gebrauchsanweisung für Portugal vorgelegt hatte, schwadroniert darin etwa davon, dass die Portugiesen »[e]in stilles, elegantes Volk« seien, freilich »gegen religiösen Eifer nicht gefeit«, und verspricht ein $»$ moderne[s] Landeskundeseminar in Form eines Buches $\ll,{ }^{8}$ das dann jedoch eher von autobiografischen Episoden wie dem ersten jugendlichen Vollrausch mit einem portugiesischen Süßwein handelt. So mündet das Kapitel »Aufbrechen « nicht etwa in eine Beschreibung der Reisewege nach Portugal, sondern in die Erkenntnis, dass »das Land, in dem ein solcher Wein wächst, [...] warm sein [musste], ein Land, in dem Milch und Honig fließen und ewige Milde herrscht. [...] Der Wunsch nach mehr Wissen über die Herkunft des köstlichen Getränks [...] war unwiderruflich geweckt. «9 Nickel bemüht sich anschließend in geradezu kolonialliterarischer Manier, die Portugiesen möglichst fremd zu machen: ein Menschenleben zähle hier nichts, es herrschten Korruption, Aberglaube, Unlogik und bürokratischer Irrsinn, die Portugiesen seien arbeitsscheu und das Land technisch rückständig. Die hierfür bemühten Beispiele - etwa der Versuch, am Straßenverkehr teilzunehmen und zu tanken - veranschaulichen die groteske Überzeichnung des Charakterbildes. Für alle Lesenden ist ersichtlich, dass hier recht banale innereuropäische Reiseerlebnisse exotisiert werden. Die penetrante Selbstinszenierung des Autors als geschmackssicherer und sensibler Dandy verbürgt, dass die Satire deutlich erkennbar ist.

Weitaus typischer für die Reihe erscheint allerdings Uli Franz' Gebrauchsanweisung für Tibet aus dem Jahr 2000. Der Autor, Verfasser zahlreicher Bücher über China und Tibet, gibt sich bereits als Alternativreisender zu erkennen, wenn er sich mit dem Rufnamen Uli vorstellt. Dass er ein Kenner der Materie ist, beglaubigt zudem ein dem Text vorangestelltes Testimonial des berühmten Bergsteigers Heinrich Harrer, Autor von Sieben Jahre in Tibet, der sich bei Franz für das »ausgezeichnete[] Buch ${ }^{10}$ bedankt. »Tibet ist kein fashionables Reiseland «, befindet Franz gleich zu Beginn,

[d]orthin zieht es einen, wenn man reif dafür ist. Dann aber gerät die Reise zur Prüfung. Nach jeder großen Reise sieht man die Welt mit anderen Augen, nach einer Tibetreise sieht man sie radikaler. [...] Tibet wird zum Fiasko für jeden, der sich nicht körperlich und mental genügend vorbereitet. Die Höhe,

\footnotetext{
8 Nickel, Eckhart: Gebrauchsanweisung für Portugal. München/Zürich ${ }^{5}$ (2010, S. 11f).

9 Nickel, Eckhart: Gebrauchsanweisung für Portugal. München/Zürich ${ }^{5}$ (2010, S. 22f).

10 Franz, Uli: Gebrauchsanweisung für Tibet. [Überarbeitete Neuausgabe] München/Zürich (2007, S. 6).
} 
die Einöde, der Wind, der Staub, die nächtliche Kälte und die Hitze des Tages nagen an der Gesundheit und erfordern beachtliche geistige Stabilität. ${ }^{11}$

Franz rät etwa davon ab, sich mit Medikamenten gegen Erschöpfung und Höhenkrankheit zu versorgen, da dem chemisch benebelten Reisenden so »die Kommunikation, die Begegnung zwischen Natur und Mensch « ${ }^{12}$ blockiert werde. Weiterhin warnt er vor »Höhenkoller« und anderen Unannehmlichkeiten: »Im schlimmsten Fall kann es zu Durchfall, Erbrechen, Schlaflosigkeit, Frieren und Sodbrennen kommen. «13 Dagegen helfe nur »richtiges Reisen «: »Rückkehr und Abschied hängen von der Art der Annäherung ab. ${ }^{14}$ Ein rascher Flug nach Lhasa mache es »unmöglich, eine Beziehung aufzubauen. Wer sich hingegen langsam, stetig an Höhe gewinnend Tibet annähert, der fühlt sich am Ende der Reise in jeder Hinsicht stärker. [...] Alle Organe haben ihre Funktion zu steigern gelernt. [...] Und der Geist tanzt, er ist beflügelt. « ${ }^{15}$ Angesichts der Fülle inhaltlicher wie sprachlicher Klischees sei darauf hingewiesen, dass es sich hierbei nicht um Satire, sondern bestenfalls Realsatire handelt. Franz würdigt die Geografie Tibets, erläutert Visafragen, Klima, Bauten, Wege, Preise, Geschichte, Religion, die politisch brisante Tibet-Frage, aber auch Ausflugsziele, Mode, Geschlechterrollen, Medizin, Sprache und Küche. Eine Besprechung der NZZ attestiert ihm dafür Ausgewogenheit: das Werk sei »differenziert[...]« und »durchweg zuverlässig «. ${ }^{16}$ Dabei finden sich durchaus eigenartige Essentialisierungen in dieser Landeskunde, wenn Franz etwa zur Veranschaulichung der Regenzeit befindet: »Die Wohnhäuser aus ungebranntem Lehm saugen sich voll und glänzen fettig. [...] Die feuchte Patina paßt gut zur dunklen Hautfarbe der Tibeter. « ${ }^{17}$ Natürlich darf auch der klassische europäische Satz über die nichteuropäischen Wilden nicht fehlen: »Tibeter sind sinnlich und verspielt wie Kinder. ${ }^{18}$ Aber auch: »Im Herzen sind alle Tibeter religiös [...]. ${ }^{19}$ Der Kollektivsingular, mithilfe dessen unterstellt wird, »der Tibeter $\aleph^{20}$ mache dies und fühle das, ist ebenfalls zu finden.

Auch Kai Strittmatters Gebrauchsanweisung für China, einem weiteren Nachbarn Nepals, konnte noch 2008 (eine frühere Fassung hatte ebenfalls der notorische Uli Franz verfasst) mit der folgenden Aussage aufwarten: »Gelb. Wollte man China auf eine Farbe reduzieren, es wäre diese. Mao Zedong mochte die >rote Sonne< sein, sein Volk blieb gelb: Gelb ist seine Haut, die Ufer des gelben Flusses sind seine Wiege, der gelbe Kaiser war sein Urahn. $\ll^{21}$ - Halten wir fest: Die Gebrauchsanweisungen

\footnotetext{
11 Franz, Uli: Gebrauchsanweisung für Tibet. [Überarbeitete Neuausgabe] München/Zürich (2007, S. 9f).

12 Franz, Uli: Gebrauchsanweisung für Tibet. [Überarbeitete Neuausgabe] München/Zürich (2007, S. 10).

13 Franz, Uli: Gebrauchsanweisung für Tibet. [Überarbeitete Neuausgabe] München/Zürich (2007, S. 10).

14 Franz, Uli: Gebrauchsanweisung für Tibet. [Überarbeitete Neuausgabe] München/Zürich (2007, S. 16).

15 Franz, Uli: Gebrauchsanweisung für Tibet. [Überarbeitete Neuausgabe] München/Zürich (2007, S. 16).

16 Lütkehaus, Ludger: »Grenzüberschreitung«. In: Neue Zürcher Zeitung 1.7.(2000, S. 68).

17 Franz, Uli: Gebrauchsanweisung für Tibet. [Überarbeitete Neuausgabe] München/Zürich (2007, S. 36f).

18 Franz, Uli: Gebrauchsanweisung für Tibet. [Überarbeitete Neuausgabe] München/Zürich (2007, S. 65).

19 Franz, Uli: Gebrauchsanweisung für Tibet. [Überarbeitete Neuausgabe] München/Zürich (2007, S. 86).

20 Franz, Uli: Gebrauchsanweisung für Tibet. [Überarbeitete Neuausgabe] München/Zürich (2007, S. 152).

21 Strittmatter, Kai: Gebrauchsanweisung für China. München/Zürich ${ }^{3}$ (2010, S. 20).
} 
des Piper-Verlages werfen sehr subjektive und als solche auch sehr deutsche Blicke auf eigene und vor allem fremde Weltteile.

Wie verortet sich nun die Gebrauchsanweisung für Kathmandu und Nepal innerhalb dieser Reihe? Exakt in der Mitte des Buches findet sich die Episode über eine Wäschereinigung in Kathmandu, einen vorbildlich organisierten Familienbetrieb, der Kracht und Nickel zufolge »[n] ur ein einziges Mal« habe »kapitulieren« müssen, und zwar angesichts der Jeanshose eines deutschen Touristen, die einen »unerhörte[n] Grad der Verschmutzung « aufwies, da der Besitzer »wohl auf der Reise schwer erkrankt « sei und sich offensichtlich »nicht geschont « habe. ${ }^{22}$ Die Hose könne man eigentlich nur noch wegwerfen, der Kassierer der Reinigung schämt sich zu sehr, um sie Kracht und Nickel zu zeigen. Er händigt ihnen jedoch ein Buch aus, »das der Deutsche bei der Abgabe seiner Kleidung in der linken hinteren Hosentasche vergessen hatte. [...] Auf dem Cover waren gerade noch so Verfasser und Titel des vergessenen Buches zu erkennen. Es handelte sich um das Werk eines geschätzten Kollegen über den hoch-infektiösen Nachbarstaat im Süden: Ilija Trojanows Gebrauchsanweisung für Indien. $\ll^{23}$

Trojanows Schilderung seiner Jahre in Indien spielen erkennbar in einer anderen Liga als Uli Franz' Tibet-Führer. Zwar geht es auch hier etwa um das herausfordernd fremde Essen, ${ }^{24}$ das als »Masala-Mix ${ }^{25}$ auch als Leitmetapher für die indische Vielfalt bemüht wird, vor allem aber erzählt Trojanow erlebte Episoden und erläutert spirituelle Konzepte, die Aspekte des indischen Kulturreichtums veranschaulichen. Das Buch und insbesondere seine Paratexte stellen heraus, dass der Autor mit den gängigen Klischees zugunsten einer Innenansicht Indiens aufräume, weil er nahe$\mathrm{zu} »$ jede Region Indiens ausgiebig bereist $\aleph^{26}$ habe und »genauer « (vordere Klappe) beziehungsweise »ganz genau« (hintere Klappe) >hinseheく. Dabei scheut mitunter auch Trojanow den Kitsch nicht, ${ }^{27}$ wenn er davon schwadroniert, dass sich die Widersprüche Indiens, »als seien die Herzen der Menschen so reich wie die Basare«, in Agra »[b]esonders eng aneinander schmiegen ${ }^{28}$ würden - Aussagen zwischen Schwulst und Nonsens, auch wenn er schreibt: »[D]ie Lärmkulisse ist eine Komposition, mit der sich die Stadt selbst fortschreibt «. ${ }^{29}$ Zudem bedient er Klischees wie die Beschwörung von »Augen [...] tief wie Brunnen « ${ }^{30}$ oder neureichen Indern als

\footnotetext{
22 Kracht, Christian/Nickel, Eckhart: Gebrauchsanweisung für Kathmandu und Nepal. München/Zürich (2009, S. 84).

23 Kracht, Christian/Nickel, Eckhart: Gebrauchsanweisung für Kathmandu und Nepal. München/Zürich (2009, S. 85).

24 Vgl. Trojanow, Ilija: Gebrauchsanweisung für Indien. München/Zürich ${ }^{3}$ (2007, S. 78ff.)

25 Trojanow, Ilija: Gebrauchsanweisung für Indien. München/Zürich ${ }^{3}$ (2007, S. 12).

26 Trojanow, Ilija: Gebrauchsanweisung für Indien. München/Zürich ${ }^{3}$ (2007, S. 12).

27 Ob dieser durch den selbstreflexiven Hinweis darauf, das Geschilderte klinge jetzt kitschig, tatsächlich gebannt werden kann, wäre zu diskutieren. Vgl. Trojanow, Ilija: Gebrauchsanweisung für Indien. München/Zürich ${ }^{3}$ (2007, S. 13).

28 Trojanow, Ilija: Gebrauchsanweisung für Indien. München/Zürich ${ }^{3}$ (2007, S. 13).

29 Trojanow, Ilija: Gebrauchsanweisung für Indien. München/Zürich ${ }^{3}$ (2007, S. 14).

30 Trojanow, Ilija: Gebrauchsanweisung für Indien. München/Zürich ${ }^{3}$ (2007, S. 32).
} 
Kinder. ${ }^{31}$ Vielleicht ist es der mit diesen exotistischen und kolonialistischen Bildern konfligierende Gestus des Insider-Experten, der nun endlich einmal jenseits westlicher Klischees das >richtige< Indien zeige, der Kracht und Nickel provoziert haben mag. ${ }^{32}$ Sicher geht es in ihrer Abgrenzung auch um Positionskämpfe im Feld der Gegenwartsliteratur. In der Mitte ihres Bandes wird auf Trojanows Gebrauchsanweisung jedenfalls buchstäblich defäkiert, was wohl eine unmissverständliche Ausstreichung auch der Piper-Reihe darstellen dürfte.

Entsprechend sind die wenigen Rezensionen professioneller Lesender von leichter Irritation und auch von Unbehagen gekennzeichnet. Burkhard Müller konstatiert in der Süddeutschen Zeitung beispielsweise, dass die Autoren den geschichtsträchtigen Ereignissen, die sie direkt miterlebt hätten, in ihren Schilderungen nicht gerecht würden: »Dem ungeheuer starken öffentlichen Affekt dieser Stunden entziehen sie sich wie eine Schnecke, der man auf die Fühlhörner tupft. Sie sind dem Moment, der ihrer hätte werden können, nicht gewachsen. $\ll^{33}$ Müller spricht weiter von "politische[r] Unbedarftheit « und fragt sich in Bezug auf die Anekdote um einen ausgerechnet deutschen Aussteiger, der als Orakel »Matthias in Lumbini« befragt wird: »Muss man für so was zehntausend Kilometer in die Ferne schweifen, von den Höhenmetern zu schweigen? «34 Im Tagesspiegel stellt Julia Boeck fest, dass die Gebrauchsanweisung von Kracht und Nickel in erster Linie unterhalten wolle, »der Blick auf die nepalesische Alltagswelt « ${ }^{35}$ aber trotzdem genau bleibe. »[D]er Bogen der Ironie«, so Boeck, werde aber teilweise überspannt und der Text bekomme so »neokoloniale Anstriche«. ${ }^{36} \mathrm{Im}$ Schweizer Monat ist der Tenor vorwiegend posi-

31 »Die aufstrebende Mittelklasse des Landes war ein Kind, das ungestüm mit Bauklötzen spielte, ohne darauf zu achten, ob es die Umgebung beschädigte.« Trojanow, Ilija: Gebrauchsanweisung für Indien. München/Zürich ${ }^{3}$ (2007, S. 46).

32 Hierfür spricht eine Besprechung von Trojanows Roman Der Weltensammler in Krachts und Nickels Magazin Der Freund, das mit Redaktionsort Kathmandu erschien. Anstatt das Buch zu besprechen, erzählen die Rezensenten die Geschichte einer kleinen Privatfehde mit Trojanow, die ihren Ausgang angeblich in einem eritreischen Restaurant nahm, in dem Trojanow sich bei einem gemeinsamen Essen als weltgewandter und kulturell beflissener Insider aufgespielt habe (vgl. Kracht, Christian/Nickel, Eckhart: »[Rez. von] Ilija Trojanow: >Der Weltensammler««. In: Der Freund 3 (2006), H. 8, S. 76f.). Die Autoren weisen in dieser >Rezension< auch auf eine Invektive gegen Trojanow hin, die sie 1998 in Ferien für immer untergebracht hatten, die im Nachdruck von 2000 absurd verändert wurde, bevor die entsprechende Passage in der Taschenbuchausgabe ab 2001 wieder in der Urfassung, aber nun mit Schwärzungen erschien. Letztere erscheinen nachträglich in der Freund $\rightarrow$ Rezension < als äquivalent zu jenen Schwärzungen, mit denen Maxim Billers mittlerweile höchstrichterlich verbotener Roman Esra noch kurzzeitig bei KiWi hatte erscheinen können (Kracht und Nickel nennen den Fall Esra ausdrücklich im Freund-Text).

33 Müller, Burkhard: »Geklauter Portwein gibt eine tolle Geschichte. Ausgewählt nach privatem Schrägheitsindex: Christian Kracht und Eckhart Nickel berichten von ihrer Zeit in Nepal«. In: Süddeutsche Zeitung 27.4.(2009, S. 14).

34 Vgl. Müller, Burkhard: »Geklauter Portwein gibt eine tolle Geschichte. Ausgewählt nach privatem Schrägheitsindex: Christian Kracht und Eckhart Nickel berichten von ihrer Zeit in Nepal«. In: Süddeutsche Zeitung 27.4.(2009, S. 14).

35 Boeck, Julia: »Die Freunde. Eine Gebrauchsanweisung für Kathmandu und Nepal«. In: Der Tagesspiegel 3.5.(2009, S. 31).

36 Boeck, Julia: »Die Freunde. Eine Gebrauchsanweisung für Kathmandu und Nepal«. In: Der Tagesspiegel 3.5.(2009, S. 31). 
tiv und die »kenntnisreichen Geschichtsdetails und Alltagsbeschreibungen Nepals« werden ganz unironisch gelobt, Kracht und Nickel seien »schliesslich Profis «. ${ }^{37}$

Die Rezensionen oder Kommentare von nicht-professionellen Leserinnen und Lesern auf amazon.de, die sich den Text zur Reisevorbereitung oder Reiselektüre gekauft haben, sind hingegen affektgeladen, oft von Empörung geprägt und geben im Vergleich zu anderen Gebrauchsanweisungen auffällig oft die niedrigst mögliche Bewertung für den Text ab. So werden beispielsweise Rezeptionserwartungen enttäuscht, die an anderen Bänden der Reihe orientiert sind. So der Kommentar mit dem Titel »Selbstbeweihräucherung und Langeweile«:

Ich habe dieses Buch als Vorbereitung für meine erste Nepalreise geschenkt bekommen. Aus der Reihe >Gebrauchsanweisung für..< [sic] besitze ich bereits die Bücher zu Island, Finnland und Spanien. Aber so eine schlechte $>\mathrm{Ge}$ brauchsanweisung für.. $<$ sic] habe ich noch nie gelesen. Die Sprache der Autoren ist sehr umständlich und bei längerer Lektüre einschläfernd. [...] Auch die ständige Werbung für die eigene Zeitung ist eher störend. Es fällt eine Überheblichkeit gegenüber Nepalesen auf [...]. Die mit Spannung und Humor erwartetend [sic] Insiderinformationen sind spärlich. Man möchte sich vom Backpack-Touristen abheben, bereist aber nur Kathmandu und Lumbini. Eine Verbindung zur Kultur und Religion (am Besten mit Erläuterungen für die Neureisenden) gibt es nicht. Das Buch ist absolut nicht empfehlenswert als Reiselektüre. $^{38}$

Die Rezeptionshaltung vieler Lesender ist also durch eine Art >paratextuelles Priming < bestimmt und auf eine literarische Wahrheitserzählung, einen Reisebericht eingestellt. Die damit verbundenen Erwartungen an den Informationsgehalt des Textes werden enttäuscht, wie auch die Rezension »Schade für's [sic] Papier « deutlich macht: »Hinter Titel und Klappentext erwartet man interessante Hintergrundinformationen zu Land und Leuten. Allerdings trifft man ein[e] wirre Sammlung von Anekdoten von 2 Westlern, die sich in erster Linie über die Nepalesen mockieren [sic]. Informationsgehalt dürftig bis nicht existent. Erstaunlich, dass ein solches Buch überhaupt einen Verleger findet... « ${ }^{39}$ Moniert werden der Mangel an nützlichen Reiseinformationen, der als wirr empfundene Aufbau, die subjektive Erzählweise und damit zusammenhängend die Inszenierung der Erzähler im Text als »2 Westler« sowie deren distanziert arrogante Haltung nepalesischen Menschen gegenüber. Wird der Text also mit den Rezeptionserwartungen an die Gebrauchsanweisungen-Reihe gelesen, sind die Irritationspotentiale erheblich, da die Peritexte, insbesondere die

\footnotetext{
37 Rottmann, Markus: »Ein Shanti von oben herab«. In: Schweizer Monat 6 (2012), H. 7, o.S. In: https:// www.schweizermonat.ch/artikel/ein-shanti-von-oben-herab (12.11.2017).

38 Rezension von »nanerine « auf amazon.de, 3.3.2013. In: https://www.amazon.de/GebrauchsanweisungKathmandu-Nepal-Christian-Kracht/dp/3492275648/ref=sr_1_2?ie=UTF8\&qid=1496051686\&sr=8-2\& keywords=gebrauchsanweisung $+\mathrm{f} \% \mathrm{C} 3 \% \mathrm{BCr}+\mathrm{kathmandu}+\mathrm{und}+$ nepal (29.5.2017).

39 Rezension von »Amazon Kunde« auf amazon.de, 17.9.2016. Ebd.
} 
Zugehörigkeit zur Reihe, eine gattungstypologische Einordnung nahelegen, die der Haupttext unterläuft. ${ }^{40}$

Sehen wir uns daraufhin den Einstieg des Bandes über Kathmandu und Nepal an. Er beginnt zunächst ganz harmlos, indem er durchaus Reihen-typisch die Autoren im fremden Land verortet. Sie führen unter anderem ihr »unstetes Dasein «, »geprägt von großen Erwartungen «, aber auch »Medikamentenabusus « und »immer wieder hastig auftretende Erleuchtungen « als Motive an, die sie nach Nepal getrieben hätten. ${ }^{41}$ Modernes Nomadentum, Drogenexperimente und Spiritualität sind nicht untypische Eigenschaften von Europäern, die es in den Himalaya zieht. Auch die Schilderung der Ankunft erscheint dabei zunächst gattungsgemäß:

Bereits beim allerersten kurzen Besuch nahm uns Nepal gefangen. Das Licht, wie können wir es beschreiben? [...] Die vielen in das Newarital fließenden Bäche ließen die gesamte Umgebung Kathmandus als überbordende Kulisse für die erhabene Freundlichkeit des nepalesischen Volkes erscheinen. Winzige Ziegelfabriken lagen über das Hochtal verstreut wie in heiliger Kinderlaune absichtslos vergessene Bauklötze. Papierne gelbe Drachen flatterten über den Dachterrassen. Kleine Grüppchen tibetanischer Mönche wichen auf dem Weg zur Stupa elegant und gelenkig den Bussen aus. Junge Hunde balgten sich im Dreck. An den Straßenrändern trocknete man ganze rote Teppiche von Chilischoten in der Sonne. ${ }^{42}$

Gängige Stereotype europäischer Reiseberichte sind hier versammelt: das angeblich besondere Licht, die »erhabene Freundlichkeit« der gleichwohl mehr oder minder subtil infantilisierten Nepalesen, auch emblematische Motive wie Drachen und Mönche werden bemüht, ebenso das gleichermaßen faszinierende wie ängstigende Nebeneinander von Tieren, Nahrungsmitteln und Schmutz auf den Straßen der sogenannten $>$ Dritten Welt $<$ : purity und danger. ${ }^{43}$

Man könnte hier schon misstrauisch geworden sein, nicht nur aufgrund der erahnbaren Überaffirmation derartiger Klischees. Warum etwa laufen tibetanische Mönche durch Nepal? Und sind nicht die nepalesischen Ziegeleien berüchtigt als Orte von Kinderarbeit und einer die Gesundheit der Arbeitenden gefährdenden Luftverschmutzung - und somit alles andere als pittoresk? Der Eindruck, es hier mit

\footnotetext{
40 Von 18 Kommentaren zur Erstausgabe (2009) geben sieben die tiefstmögliche Bewertung ab und warnen teilweise emphatisch vor dem Kauf: »Finger weg von diesem Buch« (Rezension von »Andi1978« auf amazon.de, 19.3.2012. Ebd.). Die Neuausgabe (2012) wird noch schlechter bewertet; sieben der dreizehn Bewertungen vergeben einen von fünf Sternen und warnen vor der »Geldverschwendung!!!!!!« (Rezension von »K.R.« auf amazon.de, 11.8.2014. In: https://www.amazon.de/Gebrauchsanweisung-f\%C3\%BCrKathmandu-Nepal-\%C3\%9Cberarbeitete/product-reviews/3492276156/ref=cm_cr_arp_d_paging_btm_ 2?ie=UTF8\&reviewerType=all_reviews\&pageNumber=2 [29.5.2017]).

${ }^{41}$ Kracht, Christian/Nickel, Eckhart: Gebrauchsanweisung für Kathmandu und Nepal. München/Zürich (2009, S. 13f).

${ }^{42}$ Kracht, Christian/Nickel, Eckhart: Gebrauchsanweisung für Kathmandu und Nepal. München/Zürich (2009, S. 14f).

${ }^{43}$ Vgl. Douglas, Mary: Purity and Danger. An Analysis of Concepts of Pollution and Taboo. New York (1966).
} 
den üblichen Nepalreisenden zu tun zu haben, wird im folgenden Absatz endgültig durchgestrichen:

Am dritten Tag, wir hatten gerade im uns damals noch unbekannten italienischen Restaurant >Fire and Ice< zwei Pizza Quattro Stagioni gegessen, zogen mit einemmal - es war der erste Juniabend - ungewöhnlich dräuende Monsunwolken über dem Tal von Kathmandu auf. Der Himmel färbte sich tiefrosa und orange. Die Stille war in ihrer Vollkommenheit entsetzlich. Eine schreckliche Vorahnung befiel uns zum Nachtisch - einer Schale frischer Erdbeeren mit durch die erdrückende Schwüle fast flüssig gewordener Schlagsahne. [...] In der Ferne erklang erst eine schrille Sirene, dann zwei, drei, viele. Menschen strömten auf die Straßen, sanken weinend auf die Knie, schlugen sich mit den Fäusten wehklagend an die Stirne, und die grausame Nachricht verbreitete sich in der Hauptstadt Kathmandu so rasch und schleichend wie einst Senfgas in den Schützengräben von Ypern und Malmedy. Es hieß, der Kronprinz habe erst seine gesamte Familie mit Schnellfeuergewehren erschossen und dann sich selbst. ${ }^{44}$

Auf eine vorder- wie auch hintergründige Weise beziehen Christian Kracht und Eckhart Nickel hier Opposition zu den typischen Nepal-Reisenden: zu Extrembergsteigern, Sinnsuchenden und Aussteigern. Vordergründig, weil sie eine - tatsächlich existierende - Pizzeria in Kathmandu besuchen, anstatt das Nationalgericht Dal Bhat, ein einfaches Linsengericht, zu essen und sich so der fremden Kultur auszusetzen. Der Dandy ist kein demütiger Sinnsucher, er nimmt vielmehr Erdbeeren mit Schlagsahne zum Dessert. Der Nachtisch verweist auf die hintergründige Distanzierung vom >richtigen<, alternativen Reisen, der als Kulisse der Katastrophe dienende Abendhimmel trägt ebenso wie die heulenden Sirenen dazu bei, in dieser Szene die Wiederauflage einer ganz anderen zu entdecken. Es ist jene berühmt-berüchtigte Episode aus Ernst Jüngers Zweitem Pariser Tagebuch, in der der Wehrmachtsoffizier auf dem Dach des Luxus-Hotels Raphael - »ein Glas Burgunder, in dem Erdbeeren schwammen $\ll,{ }^{45}$ in der Hand - die Bombardierung von Paris betrachtet: eine Urszene

\footnotetext{
${ }^{44}$ Kracht, Christian/Nickel, Eckhart: Gebrauchsanweisung für Kathmandu und Nepal. München/Zürich (2009, S. 15f).

45 Jünger, Ernst: Das zweite Pariser Tagebuch [Eintrag vom 27. Mai 1944]. In: Ders.: Sämtliche Werke. Tagebücher III. Strahlungen II. Stuttgart (1979, S. 9-294), hier: S. 271. - Es ist anzunehmen, dass Kracht und Nickel hier einen Vorwurf affirmieren, der einem von ihnen bereits dezidiert gemacht worden war. So fragte der Tagesspiegel in einem Interview Christian Kracht: »Der Schriftsteller Ernst Jünger hat einmal folgende Szene beschrieben: Zweiter Weltkrieg, eine Bombennacht in Paris. Er steht mit einem Glas Champagner [sic] auf dem Dach des Hotels $>$ Raphael $<$ und genießt die Ästhetik der brennenden Stadt. Wie finden Sie das? - Kracht: »Zutiefst verwerflich.« Amend, Christoph/Lebert, Stephan: »Ich weine oft<. Ist er der wahre Chronist seiner Generation? Wie blickt so einer auf die Welt? Christian Kracht ist verzweifelt und gelangweilt. Das hat ihn nach Bangkok getrieben ...«. In: Der Tagesspiegel 2.7.(2000), S. W1 [Weltspiegel].
} 
des Dandytums in der deutschen Literatur. ${ }^{46}$ So, wie schon Jüngers kalt genossene Zeugenschaft des Bombardements umstritten und wohl eher als ostentativ verrufene literarische Szene denn als authentische Erinnerung zu werten ist, ${ }^{47}$ liegt auch der Verdacht nahe, dass Kracht und Nickel während der Ermordung des Königs überhaupt nicht in Nepal waren. ${ }^{48}$

Indem Kracht und Nickel eine Schlüsselszene ihrer diversen Nepal-Aufenthalte in den zwei Jahren, in denen sie ihr Magazin Der Freund herausgaben, das sich mit dem Redaktionsort Kathmandu schmückte, als Parodie einer unschwer zu dechiffrierenden literarischen Szene anlegen - als deutlichen Hinweis, um das Ernst Jünger-Zitat bei den Lesenden zu triggern, schieben sie den Vergleich mit dem »Senfgas in den Schützengräben von Ypern und Malmedy« nach -, verletzen sie den >reiseliterarischen Pakt< von going und knowing. Schlagartig erscheinen auch andere Bilder, die sie entwerfen, als intertextuelle Verweise, etwa jene »Teppiche von Chilischoten «, die auch aus dem Comicalbum Tim in Tibet stammen könnten ${ }^{49}$ - wie auch die angeblich »von Nepalis zum Essen gerne bestellte 1,5 Liter Flasche Whisky $\ll^{50}$, im Universum von Tim und Struppi der Treibstoff von Tims ständigem Begleiter Kapitän Haddock. Der Band, der auch Kathmandu berührt, wird in der Gebrauchsanweisung explizit erwähnt. ${ }^{51}$

Das Eingangskapitel veranschaulicht, wie die Autoren eine Erwartungsfalle aufbauen, indem die der Gattung Reiseliteratur gemäßen Erwartungen im weiteren Verlauf andauernd unterminiert werden. Sie geben keine nützlichen Tipps, würdi-

46 Vgl. dazu Kiesel, Helmuth: Ernst Jünger. Die Biographie. München (2007, S. 518ff. -) Kiesel fasst zusammen: »Unzählige Male ist diese Stelle als Beispiel für Jüngers einzigartigen amoralischen oder gar barbarischen Ästhetizismus angeführt, als skandalös bezeichnet und als kitschig verurteilt worden.« (Ebd., S. 518.) Der ganze Eintrag, dessen ursprüngliche Fassung Jünger mit erkennbarem Stilwillen mehrfach bearbeitet hat (vgl. dazu ebd.), lautet: »Alarme, Überfliegungen. Vom Dache des >Raphael< sah ich zweimal in Richtung von Saint-Germain gewaltige Sprengwolken aufsteigen, während Geschwader in großer Höhe davonflogen. Ihr Angriffsziel waren die Flußbrücken. Art und Aufeinanderfolge der gegen den Nachschub gerichteten Maßnahmen deuten auf einen feinen Kopf. Beim zweiten Mal, bei Sonnenuntergang, hielt ich ein Glas Burgunder, in dem Erdbeeren schwammen, in der Hand. Die Stadt mit ihren roten Türmen und Kuppeln lag in gewaltiger Schönheit, gleich einem Kelche, der zu tödlicher Befruchtung überflogen wird. Alles war Schauspiel, war reine, von Schmerz bejahte und erhöhte Macht.« Jünger, Ernst: Das zweite Pariser Tagebuch [Eintrag vom 27. Mai 1944]. In: Ders.: Sämtliche Werke. Tagebücher III. Strahlungen II. Stuttgart (1979, S. 271).

47 Vgl. Wimbauer, Tobias: »Kelche sind Körper. Der Hintergrund der >Erdbeeren in Burgunder<-Szene«. In: Ders. (Hg.): Anarch im Widerspruch. Neue Beiträge zum Werk und Leben der Gebrüder Jünger. Schnellroda (2004, S. 23-69). -) Wimbauer weist nach, dass an besagtem Tag gar kein Luftangriff auf Paris geflogen wurde.

48 2001, als sich das Royal Massacre in Nepal ereignete, übersiedelte Kracht jedenfalls gerade von Bangkok nach Sri Lanka; es ist zwar theoretisch möglich, dass er sich im Sommer noch 2001 im tibetischen Lhasa befand, wo er seinen im gleichen Herbst erschienen Roman 1979 schrieb - in Nepal kam er jedenfalls erst 2003 an (wenn man dem biografischen Überblick über Reisen und Wohnorte Krachts im Sammelband von Johannes Birgfeld und Claude D. Conter Glauben schenken darf - vgl. Dies. (Hrsg.): Christian Kracht. Zu Leben und Werk. Köln (2009, S. 271-278).).

49 Vgl. Hergé: Tim in Tibet (= Tim und Struppi, Bd. 19). Hamburg (1999, S. 13).

${ }^{50}$ Kracht, Christian/Nickel, Eckhart: Gebrauchsanweisung für Kathmandu und Nepal. München/Zürich (2009, S. 54).

${ }^{51}$ Vgl. Kracht, Christian/Nickel, Eckhart: Gebrauchsanweisung für Kathmandu und Nepal. München/ Zürich (2009, S. 20). 
gen keine touristischen Highlights oder das lokale Essen, stattdessen spüren sie in Antiquariaten den Spuren der Hippies nach und kaufen ausgerechnet deutsche Bücher auf. Beschreiben sie einmal Nepalesen, dann klassifizieren sie diese klar in einem eurozentrischen Referenzrahmen, indem sie diese als »der nepalesische Samuel Beckett«, »der Siegfried Krakauer Nepals« oder »eine indische Version von Walter Benjamin « vorstellen. ${ }^{52}$ Eine Kippfigur im Wortsinne ergibt sich in der Verschreibung des Namens von Siegfried Kracauer als »Krakauer«, ruft der Name in dieser Schreibweise im nepalesischen Kontext doch eher Jon Krakauer auf, Autor des Bestsellers Into thin air (1997; bei Piper 1998 als In eisigen Höhen erschienen), indem dieser das tödliche Scheitern einer rein kommerziellen, touristischen EverestBesteigung beschreibt. Jede Bewegung dieses >Reisens< in Anführungszeichen endet in der Bezugnahme auf Literatur, wobei die offensichtlichsten Fährten, die die Autoren legen, wiederum vordergründig in die Irre führen.

So handelt die von Kracht und Nickel für den Hippie-Strom der 60er Jahre verantwortlich gemachte Morgenlandfahrt Hermann Hesses ${ }^{53}$ mitnichten vom Orient, sondern erschöpft sich weitgehend in einer »todesmutigen Durchquerung Oberschwabens $«,{ }^{54}$ sie erreicht bestenfalls das Tessin, eventuell noch Italien. ${ }^{55}$ Hintergründig verbindet jedoch die Autoren der Gebrauchsanweisung mit Hesses Protagonist »H. H. « ihre kulturkonservative Ablehnung »moderner Dutzendreisen $\ll{ }^{56} \mathrm{H}$. H. scheitert schließlich bei der Wiedergabe seiner Reiseerlebnisse und muss erkennen, dass »die erdachten Figuren [...] viel lebendiger, schöner, froher und gewissermaßen richtiger und wirklicher als die Dichter und Schöpfer selber « ${ }^{57}$ wirken. Sein spiritueller Meister belehrt ihn: »Gerade das ist es ja, das Leben, wenn es schön und glücklich ist: ein Spiel! $\ll^{58}$ Das intertextuelle Spiel enthüllt den informierten Lesenden der Gebrauchsanweisung den Charakter ihrer Lektüre, es sind Fiktionalitätsmarker und damit Leseanweisungen: Die Einspielung Hesses weist eben gerade nicht nach Osten, sondern zurück in die Literatur.

Der Authentizitätsanspruch wird nicht nur intertextuell, sondern auch auf anderen Ebenen des Textes unterlaufen. Zwar wird im Klappentext eine Situation unmittelbarer Augenzeugenschaft des politischen Umbruchs in Nepal behauptet: »Von ihrem Balkon am Durbar Square beobachteten die beiden Autoren im Mai 2008 den Ab-

\footnotetext{
52 Kracht, Christian/Nickel, Eckhart: Gebrauchsanweisung für Kathmandu und Nepal. München/Zürich (2009, S. 34f., 42).

53 Vgl. Kracht, Christian/Nickel, Eckhart: Gebrauchsanweisung für Kathmandu und Nepal. München/ Zürich (2009, S. 22).

54 Hesse, Hermann: Die Morgenlandfahrt. Eine Erzählung. In: Ders.: Gesammelte Dichtungen. Sechster Band. Frankfurt am Main/Zürich (1952, S. 38).

55 Vgl. Hesse, Hermann: Die Morgenlandfahrt. Eine Erzählung. In: Ders.: Gesammelte Dichtungen. Sechster Band. Frankfurt am Main/Zürich (1952, S. 23).

56 Mit »Eisenbahnen, Dampfschiffe[n], Telegraph, Auto, Flugzeug« (Hesse, Hermann: Die Morgenlandfahrt. Eine Erzählung. In: Ders.: Gesammelte Dichtungen. Sechster Band. Frankfurt am Main/Zürich (1952, S. 10)).

57 Hesse, Hermann: Die Morgenlandfahrt. Eine Erzählung. In: Ders.: Gesammelte Dichtungen. Sechster Band. Frankfurt am Main/Zürich (1952, S. 27).

58 Hesse, Hermann: Die Morgenlandfahrt. Eine Erzählung. In: Ders.: Gesammelte Dichtungen. Sechster Band. Frankfurt am Main/Zürich (1952, S. 49).
} 
lauf der ersten freien Wahlen in Nepal. « ${ }^{59}$ Diese scheinbare Realitätsreferenz auf ein konkretes historisches Ereignis wird allerdings fragwürdig, da diese Wahlen nicht im Mai sondern bereits am 10. April 2008 stattgefunden haben. ${ }^{60}$ Dadurch wird der Realitätseffekt durchkreuzt und die scheinbare Referenz auf ein reales Ereignis wird zu einem Fiktionalitätssignal umcodiert. Die Zuverlässigkeit der Erzählinstanz wird also bereits im Klappentext in ein zweifelhaftes Licht gerückt. Dass sich die Autoren verweigern, den Rezeptionserwartungen an einen Reiseführer gerecht zu werden, stellen sie in der Einleitung offen aus: »Daß das Base Camp des Everest vermüllt ist, die Überzahl der Nepalis erschreckend arm, Kathmandu von Smog und Abgasen eingehüllt, wollen wir Ihnen nicht erzählen, diese Wahrheiten sind im Internet schnell und beliebig zu erfahren. ${ }^{61}$ Anstatt der wissensvermittelnden Funktion von Reiseführern gerecht zu werden und nützliche Informationen und Reisehinweise zu geben, rufen sie die Lesenden implizit zur eigenständigen Internetrecherche auf. So wird klar, dass es den Autoren nicht um die Vermittlung solcher >Wahrheiten $<$ geht, was im darauffolgenden Satz erneut ausgestellt wird: »Statt dessen haben wir uns bemüht, Ihnen das Kaleidoskop dieses winzigen, wundervollen Landes, dessen Reinheit in seiner Seele wohnt und das sieben der zehn höchsten Berge unseres Planeten beherbergt, so zu zeigen, wie es uns vergönnt war hineinzuschauen: Namasté. ${ }^{62}$ Hier irritiert nicht nur die esoterisch anmutende, überdrehte Rhetorik, sondern auch der manifeste Fehler von sieben anstatt acht der zehn höchsten Berge; ein faktischer Fehler also, der der Empfehlung im vorherigen Satz folgend »im Internet schnell und beliebig « in Erfahrung gebracht werden könnte. Die faktisch korrekte Zahl Acht wurde hier durch die symbolisch aufgeladene Zahl Sieben ersetzt, die in diesem Kontext als Anspielung auf Schneewittchen und die sieben Zwerge hinter den sieben Bergen und somit als Hinweis auf die Märchenhaftigkeit der Erzählung gedeutet werden kann oder auch als Anklang an Heinrich Harrers Sieben Jahre in Tibet. ${ }^{63}$ Auf die >uneigentliche< Erzählweise der Gebrauchsanweisung deutet auch die in der Einleitung etablierte Verbindung zu den ebenfalls von Kracht und Nickel

\footnotetext{
59 Kracht, Christian/Nickel, Eckhart: Gebrauchsanweisung für Kathmandu und Nepal. München/Zürich (2009), Klappentext.

$60 »$ On 10 April, 2008 Nepal held wholly unprecedented and epochal nationwide elections - the most peaceful in its history«. Hachhethu, Krishna/Gellner, David N.: »Nepal. Trajectories of democracy and restructuring of the state «. In: Paul R. Brass (Hg.): Routledge Handbook of South Asian Politics. India, Pakistan, Bangladesh, Sri Lanka, and Nepal. London/New York (2010, S. 131-146), hier: S. 131. Vgl. Shrestha, Nanda R./Bhattararai, Keshav: Historical Dictionary of Nepal. Lanham u.a. ${ }^{2(2017)}$ (= Historical Dictionaries of Asia, Oceania, and the Middle East), S. xxxvii, xlv.

61 Kracht, Christian/Nickel, Eckhart: Gebrauchsanweisung für Kathmandu und Nepal. München/Zürich (2009, S. 17).

62 Kracht, Christian/Nickel, Eckhart: Gebrauchsanweisung für Kathmandu und Nepal. München/Zürich (2009, S. 18).

63 Harrer, Heinrich: Sieben Jahre in Tibet. Mein Leben am Hofe des Dalai Lama. Wien (1952).
} 
in Co-Autorschaft verfassten fiktiven Reiseminiaturen Ferien für immer ${ }^{64}$ (1998) hin. Die Idee dazu, nach Nepal zu reisen, hatten die Autoren nach eigener Angabe »[a]uf dem Friedhof von Banda Neira $«,{ }^{65}$ dem die Miniatur »maulana inn, bandaneira indonesien ${ }^{66}$ in Ferien für immer gewidmet ist und die Beschreibung, dass die Bewohner ihre Blue Jeans auf den Grabsteinen trocknen würden, überlagern sich in beiden Texten. ${ }^{67}$

Auf einen Modus > uneigentlichen Sprechens $<$ macht auch aufmerksam, dass geläufige, klischeehafte Sprachbilder wie die des >Daches der Welt< bei Kracht und Nickel mit ironisierendem Gestus weitergeführt werden, sodass in grotesker Überbietung aus Nepal »das Land am Giebelfirst des Daches der Welt « ${ }^{68}$ wird.

In konsequenter Folge der in Klappentext und Einleitung etablierten Unzuverlässigkeit und >Uneigentlichkeit< erzählt das zweite Kapitel, »Wege nach Nepal«, eine kontrafaktische Ereignisgeschichte. Die Behauptung, dass es sich bei Nepal um ein »leider eisenbahnlos gebliebene[s] Land $\ll{ }^{69}$ handle, erweist sich beispielsweise als falsch und die anekdotenhafte Schilderungen des gescheiterten Kolonisierungsversuchs als hochgradig zweifelhaft. Der Landweg nach Nepal, so die Autoren, trage »immer noch den Makel des verzweifelten Versuches der britischen Armee, im neunzehnten Jahrhundert Nepal zu erobern. $^{70}$ In der Version der Gebrauchsanweisung scheiterte dieses Vorhaben an der Malaria übertragenden Stechmücke, die eine Überwindung der Mahabharat-Kette verunmöglicht und unzählige Todesfälle

64 Kracht, Christian/Nickel, Eckhart: Ferien für immer. Die angenehmsten Orte der Welt. [Mit einem Vorwort von Moritz von Uslar] Köln (1998). Auf den fiktiven Charakter und die intertextuelle Verfasstheit der Reiseminiaturen hat Matthias N. Lorenz anhand der Miniatur »carolina guest house hingewiesen. Vgl. Lorenz, Matthias N.: »>Au weia. Kein Frühstück ohne Papaya.< Poetiken des Pazifiks bei Christian Kracht und Hans Christoph Buch«. In: Johannes Görbert/Mario Kumekawa/Thomas Schwarz (Hg.): Pazifikismus. Poetiken des Stillen Ozeans. Würzburg (2017) (= Rezeptionskulturen in Literatur- und Mediengeschichte, Bd. 8), S. 461-479.

65 Kracht, Christian/Nickel, Eckhart: Gebrauchsanweisung für Kathmandu und Nepal. München/Zürich (2009, S. 14).

66 Kracht, Christian/Nickel, Eckhart: Ferien für immer. Die angenehmsten Orte der Welt. [Mit einem Vorwort von Moritz von Uslar] Köln (1998, S. 83-85).

67 Vgl. Kracht, Christian/Nickel, Eckhart: Ferien für immer. Die angenehmsten Orte der Welt. [Mit einem Vorwort von Moritz von Uslar] Köln (1998, S. 85); vgl. Kracht, Christian/Nickel, Eckhart: Gebrauchsanweisung für Kathmandu und Nepal. München/Zürich (2009, S. 14).

68 Kracht, Christian/Nickel, Eckhart: Gebrauchsanweisung für Kathmandu und Nepal. München/Zürich (2009), Klappentext, vgl. S. 23.

69 Kracht, Christian/Nickel, Eckhart: Gebrauchsanweisung für Kathmandu und Nepal. München/Zürich (2009, S. 19). Diese Behauptung wird in der Gebrauchsanweisung ein weiteres Mal gemacht: »in Nepal, ganz ähnlich wie auf Barbados oder im Gazastreifen, gibt es leider keinen einzigen Eisenbahnkilometer« (ebd., S. 99). Tatsächlich wurde die erste Eisenbahnverbindung der Nepal Government Railway nach Indien jedoch bereits 1928 gebaut und eine zweite Strecke wurde 1937 eröffnet. Beide Strecken befinden sich in schlechtem Zustand, sind aber nach wie vor in Gebrauch. Vgl. Shrestha, Nanda R./Bhattararai, Keshav: Historical Dictionary of Nepal. Lanham u.a. ${ }^{2}$ 2017) (= Historical Dictionaries of Asia, Oceania, and the Middle East), S. 278. Siehe dazu auch: Whelpton, John: A History of Nepal. Cambridge (2005, S. 138): »Roads were complemented by three other transportation systems. In 1951 there had been two stretches of railway line in operation, crossing the border between Raxaul and Birgani and between Jaynagar and Janakpur.«.

70 Kracht, Christian/Nickel, Eckhart: Gebrauchsanweisung für Kathmandu und Nepal. München/Zürich (2009, S. 20). 
verursacht habe: »Nepal blieb von den Briten und allen anderen Fremdmächten uneingenommen, das Gebirge wird im Volksmund seitdem auch gern Malariabarata genannt. $\ll^{71}$

Diese unglaubwürdige Anekdote wird anstelle des Anglo-nepalesischen Krieges (1814-1816) erzählt, der in der Chronologie komplett ausgespart bleibt. Der Anglonepalesische Krieg, auch Gurkha-Krieg genannt, war eine militärische Auseinandersetzung zwischen dem Gurkha-Königreich im Norden Nepals und der britischen Ostindien-Kompanie. Der Konflikt wurde durch die expansive Politik der Gurkha ausgelöst, die im Süden auf den Einflussbereich der britischen Ostindien-Kompanie trafen und entbrannte infolgedessen um die Kontrolle der Grenzgebiete und die Aushandlung der Grenzziehung. ${ }^{72}$ Der Vertrag von Sigauli, der diesen Krieg beendete, wird in der Chronologie der Gebrauchsanweisung zwar genannt, allerdings wurde dieser nicht wie behauptet 1810 , sondern 1816 geschlossen. ${ }^{73}$ Durch diese fehlerhafte Ereignisgeschichte Nepals inszenieren Kracht und Nickel, die eine »fast erschreckende[ ] Renitenz des kleinen Volkes im Himalaja « ${ }^{74}$ konstatieren, Nepal in der Art des wehrhaften und uneinnehmbaren gallischen Dorfes der Asterix und Obelix-Comics und behaupten, es seien die Bestrebungen des Empires gewesen »einen weiteren, britisch besetzten Pufferstaat zwischen Indien und das zaristische Rußland zu schieben $\ll^{75}$, obwohl Nepal im Norden an Tibet und China grenzt.

Dem als gescheitert beschriebenen Kolonialvorhaben der Briten im frühen 19. Jahrhundert stellen Kracht und Nickel die in den 60er und 70er Jahren über den so genannten Hippie-Trail nach Nepal Reisenden als die wahre usurpatorische Kraft gegenüber. Der >Einfall $<$ der Hippies wird als erodierende Naturgewalt geschildert und das Vordringen der Hippies ab 1966 als »Rinnsal« und schließlich als >Dammbruch< beschrieben. ${ }^{76}$ Die Reisepraxis der Hippies und deren kulturelle Appropriationspraktiken werden als (neo-)kolonial-schmarotzerhaftes Gebaren beschrieben, aus dem ein, so die Autoren, »im Sinne Edward Saids zu verstehender Orientalismus erwuchs; der der Trekker. «77 Alternativreisende und Rucksacktouristinnen und -touristen, die wohl einen Großteil des Zielpublikums der »Gebrauchsanweisungen«-Reihe ausmachen, werden also direkt in diese Traditionslinie gestellt, der Text

\footnotetext{
71 Kracht, Christian/Nickel, Eckhart: Gebrauchsanweisung für Kathmandu und Nepal. München/Zürich (2009, S. 21). Das Wortspiel verweist auf das indische Epos Mahabharata, das Kracht in seiner Dankesrede zur Verleihung des Wilhelm-Raabe-Literaturpreises 2012 als Inspiration genannt hat und das auch in Die Toten (2016) genannt wird. Kracht, Christian: Die Toten. Roman. Köln (2016, S. 138).

72 Vgl. Shrestha, Nanda R./Bhattararai, Keshav: Historical Dictionary of Nepal. Lanham u.a. ${ }^{2(2017)(=}$ Historical Dictionaries of Asia, Oceania, and the Middle East), S. xxix.

73 »The Treaty of Sagauli (Sigauli) ratified in March [1816]. As part of the treaty, Nepal ceded roughly one-half of its territory to British India. «Shrestha, Nanda R./Bhattararai, Keshav: Historical Dictionary of Nepal. Lanham u.a. ( ${ }^{2}$ 2017) (= Historical Dictionaries of Asia, Oceania, and the Middle East), S. xxix.

74 Kracht, Christian/Nickel, Eckhart: Gebrauchsanweisung für Kathmandu und Nepal. München/Zürich (2009, S. 21).

75 Kracht, Christian/Nickel, Eckhart: Gebrauchsanweisung für Kathmandu und Nepal. München/Zürich (2009, S. 21).

76 Vgl. Kracht, Christian/Nickel, Eckhart: Gebrauchsanweisung für Kathmandu und Nepal. München/ Zürich (2009, S. 24).

77 Kracht, Christian/Nickel, Eckhart: Gebrauchsanweisung für Kathmandu und Nepal. München/Zürich (2009, S. 25).
} 
wendet sich somit explizit gegen einen Großteil der vom Verlag und Reihenlayout angesprochenen Zielgruppe. Die Irritation der Rezipierenden, wie sie sich in den erbosten amazon-Kommentaren äußert, ist im Text durch eine Art Publikumsbeschimpfung sowie durch die Verschiebung und Verfälschung historischer Ereignisse angelegt.

Neben dieser kontrafaktischen Geschichtsschreibung Nepals und der geografischen Irritation lassen sich zahlreiche weitere Fehler, wie falsch geschriebene Namen realexistierender Personen, ausmachen. ${ }^{78}$ Dieses Irritationspotential lässt das Referenzverhältnis zur außersprachlichen Realität und zu den historischen Begebenheiten fragwürdig werden und macht deutlich, dass es sich hierbei um Fiktionalitätssignale handelt. ${ }^{79}$

Obwohl sie als solche einen Authentizitätsanspruch des Textes untergraben, werden ihnen paratextuell wiederum Beglaubigungsstrategien gegenübergestellt. Die in dem Kapitel $\gg$ Der steile Aufstieg und Fall der Hermann-Hesse-Gesellschaft ${ }^{80}$ geschilderte Veranstaltung in den Redaktionsräumen der Zeitschrift Der Freund wird beispielsweise über zahlreiche Informationen mit dokumentarischem Charakter auf der Facebook-Seite und der Website von Der Freund beglaubigt - so beispielsweise Fotos der Autoren in Kathmandu, ein Scan der Einladung zum beschriebenen Anlass, eine Quittung des Hotel Sugat oder die im Band selbst abgedruckten Artikel nepalesischer Zeitungen, die über den Anlass berichten. ${ }^{81}$

Durch teilweise geringe Abweichungen zwischen den (pseudo-)dokumentarischen Materialien und der Gebrauchsanweisung wird das verzerrte Referenzsystem erneut sichtbar: So ist beispielsweise das Goethe Zentrum Kathmandu auf der Einladung als Kooperationspartner aufgeführt, obwohl das Zentrum den Anlass der Gebrauchsanweisung zufolge nicht unterstützt hat. ${ }^{82}$ Dieser Sachverhalt wird in einem auf der Website von Der Freund veröffentlichten Brief wieder aufgenommen, den der Direktor des Goethe Zentrum Kathmandu, Michael Chand, datiert auf den 2. August 2004, mit einer Aufforderung zur Klärung an die Freund-Redaktion geschickt

\footnotetext{
78 Bspw. »Ramesh Adikhari« statt Ramesh Adhikari, »Siegfried Krakauer« statt Siegfried Kracauer, »David Woodward« statt David Woodard. Kracht, Christian/Nickel, Eckhart: Gebrauchsanweisung für Kathmandu und Nepal. München/Zürich (2009, S. 28, 35, 111).

${ }^{79}$ Auf diese Poetik der Irritation angesprochen, hat Krachts Verleger Helge Malchow im Interview mit Christoph Kleinschmidt für den 2017 erschienenen Text+Kritik-Band Folgendes konstatiert: »Man kann bei der Lektoratsarbeit Christian Kracht zur Weißglut bringen, wenn man ihn darauf hinweist, dass irgendein historisches Ereignis >nicht stimmt «.« Malchow, Helge/Kleinschmidt, Christoph: »Hermeneutik des Bruchs oder Die Neuerfindung frühromantischer Poetik. Ein Gespräch«. In: Christoph Kleinschmidt (Hg.): Text+Kritik. Zeitschrift für Literatur. Christian Kracht (2017), H. 216, S. 34-43, hier: S. 37.

${ }^{80}$ Kracht, Christian/Nickel, Eckhart: Gebrauchsanweisung für Kathmandu und Nepal. München/Zürich (2009, S. 28-51).

${ }^{81}$ Abzüge der zitierten Zeitungsartikel der nepalesischen Presse, die über den Anlass berichten, finden sich auf der Website von Der Freund in der Rubrik »Presse«, in der auffälligerweise ausschließlich Berichte nepalesischer Zeitungen aufgeführt sind und die zahlreichen Ankündigungen und Rezensionen deutschsprachiger Feuilletons fehlen. Vgl. Website Der Freund, Rubrik »Presse«. In: http://www.derfreund. com/presse.php (13.11.2017). Der Scan der Einladung wurde am 6.11.2015 auf der Facebook-Seite von Der Freund gepostet. In: https://www.facebook.com/der.freund.magazine/photos/a.10151583442527445. 1073741826.54088502444/10153692584962445/?type=3\&theater. (12.11.2017).

82 Vgl. Kracht, Christian/Nickel, Eckhart: Gebrauchsanweisung für Kathmandu und Nepal. München/ Zürich (2009, S. 31).
} 
hat. ${ }^{83}$ Dieses Dokument widerspricht dem Szenario in der Gebrauchsanweisung, in dem »der furchtbare Direktor des Goethe-Zentrums $«,{ }^{84}$ wenn auch nicht namentlich benannt, an der Veranstaltung teilnimmt. Diese Materialien mit scheinbar dokumentarischem und beglaubigendem Charakter verstärken durch Abweichungen die Unzuverlässigkeit der Erzählung und deuten auf ein verzerrtes Referenzverhältnis zur außersprachlichen Realität hin.

In Bezug auf diese Irritationspotentiale erweist sich ein Abgleich zwischen der Erstausgabe und der überarbeiteten Neuausgabe von 2012 als ausgesprochen aufschlussreich. ${ }^{85}$ Ähnlich wie die von Kracht und Nickel verfassten Reiseminiaturen in Ferien für immer, die ebenfalls in einer überarbeiteten und ergänzten Neuausgabe erschienen sind, weist auch die Neuausgabe der Gebrauchsanweisung signifikante Abweichungen zur Erstausgabe auf.

Die Dichte der Änderungen - Korrekturen, Streichungen und Ergänzungen ist in den Peritexten besonders hoch. Beispielsweise wurde das für die Reihe ungewöhnliche schwarz-weiße Titelbild der Erstausgabe, das einen westlichen Reisenden zeigt, durch eine hinsichtlich der gestalterischen Konventionen der Reihe konformere, farbige Fotografie der Stupa bei Pangboche und der dahinter liegenden Bergansicht ersetzt. ${ }^{86}$ Auch in den Klappentexten wurden auffällig viele Änderungen vorgenommen. Bereits im ersten Satz des vorderen Klappentextes wurde die Anzahl der höchsten Berge nun von sieben auf acht korrigiert. ${ }^{87}$ Der Superlativ der »höchste[n] Tempeldichte der Erde ${ }^{88}$, ein Attribut, das gemeinhin eher der indonesischen Insel Bali oder den kambodschanischen Tempelanlagen um Angkor Wat zugeschrieben wird, ist durch die vorsichtigere Formulierung einer »beeindruckende[n] Tempeldichte $\ll^{89}$ ersetzt, die sich der faktischen Überprüfbarkeit entzieht. Aus dem Klappentext gestrichen wurde der Satz, dass, wer mit den Autoren nach Nepal reise, »Tee mit dem maoistischen Premierminister Prachanda trinke $\ll^{90}$, der sich auf

\footnotetext{
83 Micheal D. Chand Thakuri war von 2000 bis 2015 Direktor des Goethe-Instituts Kathmandu, und ihm wurde 2008 von Horst Köhler das Bundesverdienstkreuz für die Förderung deutscher Sprache und Kultur in Nepal verliehen. Vgl. Website des Projekts »Poets translating Poets. Poetry traverses South Asia and Germany«, einer Kooperation des Goethe-Instituts in Kooperation mit der Deutschen UNESCO-Kommission. In: http://www.goethe.de/ins/in/lp/prj/ptp/tra/en15346004.htm (12.11.2017).

84 Kracht, Christian/Nickel, Eckhart: Gebrauchsanweisung für Kathmandu und Nepal. München/Zürich (2009, S. 42).

85 Kracht, Christian/Nickel, Eckhart: Gebrauchsanweisung für Kathmandu und Nepal. [Überarbeitete und erweiterte Neuausgabe] München/Zürich (2012).

86 Unter den Gebrauchsanweisungen gibt es abgesehen von der Erstausgabe der Gebrauchsanweisung für Kathmandu und Nepal keinen Band mit schwarz-weißer Fotografie auf dem Buchcover. Auf das Motiv der Erstausgabe wird am Ende des Beitrags noch ausführlich Bezug genommen.

87 »Nepal! Das Land am Giebelfirst des Daches der Welt, wo sieben [acht] der zehn höchsten Berge in den Himmel ragen.« (Kracht, Christian/Nickel, Eckhart: Gebrauchsanweisung für Kathmandu und Nepal. München/Zürich (2009, 2012), vorderer Klappentext.) Die faktisch falsche Angabe wurde auch am Ende der Einleitung korrigiert (Vgl. 2009, S. 18; 2012, S. 17).

88 Kracht, Christian/Nickel, Eckhart: Gebrauchsanweisung für Kathmandu und Nepal. München/Zürich (2009), Klappentext.

89 Kracht, Christian/Nickel, Eckhart: Gebrauchsanweisung für Kathmandu und Nepal. [Überarbeitete und erweiterte Neuausgabe] München/Zürich (2012), Klappentext.

90 Kracht, Christian/Nickel, Eckhart: Gebrauchsanweisung für Kathmandu und Nepal. München/Zürich (2009), Klappentext.
} 
das Kapitel »Ein Tee mit Prachanda ${ }^{91}$ bezieht, in dem ähnlich wie in dem Kapitel »Matthias in Lumbini «92 ein wohl fiktives Interview wiedergegeben wird. Der Verweis auf diese fiktive Passage, die sich im Gegensatz zu »Matthias in Lumbini« mit einer real existierenden Person des öffentlichen Lebens befasst, wurde also wahrscheinlich da falsifizierbar - gelöscht..$^{93}$

Eine weitere Korrektur wurde im hinteren Klappentext vorgenommen, in dem das Text-Bild-Verhältnis zwischen Autorenfoto und Bildunterschrift vereindeutigt wurde: In der Erstausgabe sind die abgebildeten Autoren in irritierender Weise verkehrt herum ausgewiesen, sodass Lesende, die mit den Autoren nicht vertraut sind, Eckhart Nickel als Christian Kracht identifizieren und umgekehrt. In der überarbeiteten Neuausgabe wird der Bezug durch die Ergänzung »Christian Kracht (r.)《 eindeutig zugewiesen.

Neben dieser Korrektur wurde im hinteren Klappentext auch eine signifikante Streichung vorgenommen. Der bereits erwähnte Satz: »Von ihrem Balkon am Durbar Square beobachteten die beiden Autoren im Mai 2008 den Ablauf der ersten freien Wahlen in Nepal. $\ll^{94}$ wurde ersatzlos entfernt, und es liegt nahe anzunehmen, dass die Streichung darauf zurückzuführen ist, dass diese Angabe faktisch falsch ist. Zugleich weist die behauptete authentische, unmittelbare Beobachtung die Erzählung als unzuverlässig aus.

Obwohl die Anfragen bezüglich Änderungen zwischen Erst- und Neuausgabe durch den Piper-Verlag bis dato nicht beantwortet wurden, drängt sich der Verdacht auf, dass die Klappentexte unter Einflussnahme des Verlags normalisiert wurden, indem Irritationspotentiale wie manifeste Fehler korrigiert (etwa die Anzahl der höchsten Berge), potenziell Missverständliches klargestellt (wie die Identifikation der Autoren), und Hinweise auf den weitgehend fiktionalen Charakter des Textes gestrichen wurden (wie die >Lüge< der Augenzeugenschaft). Während die Gebrauchsanweisung für Kathmandu und Nepal in den Peritexten der Neuausgabe weitgehend >entstört < und wieder in das Ordnungssystem der Reihe integriert wurde, zeigen die Änderungen im Haupttext in eine andere Richtung, denn der hohe Grad an Literarizität wird noch stärker ausgestellt - insbesondere wenn beide Ausgaben vergleichend gelesen werden, wie das Beispiel der folgenden Ergänzung verdeutlicht: Es handelt sich um das Interview mit Matthias in Lumbini, das bereits in der Erstausgabe zahlreiche Fiktionalitätssignale aufweist, die in der Neuausgabe noch verstärkt werden. In die Interviewpassage wurde beispielsweise folgende Beschreibung eingefügt: »Ganz kurz ziehen sich seine Mundwinkel zusammen, als habe er sich erneut in eine bereits offene Stelle auf der Zunge gebissen. Dann lächelt er

\footnotetext{
${ }^{91}$ Kracht, Christian/Nickel, Eckhart: Gebrauchsanweisung für Kathmandu und Nepal. München/Zürich (2009, S. 139-160).

92 Kracht, Christian/Nickel, Eckhart: Gebrauchsanweisung für Kathmandu und Nepal. München/Zürich (2009, S. 86-94).

93 Pushpa Kamal Dahal, genannt Prachanda, war von August 2008 bis Mai 2009 Premierminister von Nepal und ist dies erneut seit August 2016. Vgl. Shrestha, Nanda R./Bhattararai, Keshav: Historical Dictionary of Nepal. Lanham u.a. ${ }^{2}(2017)$ (= Historical Dictionaries of Asia, Oceania, and the Middle East), S. xlv.

${ }^{94}$ Kracht, Christian/Nickel, Eckhart: Gebrauchsanweisung für Kathmandu und Nepal. München/Zürich (2009), hinterer Klappentext.
} 
uns offen an, von schräg unten. $\ll^{95}$ Diese Ergänzung von Mikroexpressionen, die in Interviews häufig als Stilmittel eingesetzt wird, um interviewte oder portraitierte Personen plastischer und lebensnäher zu beschreiben, ${ }^{96}$ suggeriert als Authentizitätsmarker einerseits einen direkten Realitätsbezug, eine genaue Beobachtung und Beschreibung. Vergleicht man die Ausgaben aber, gewinnt die Ergänzung den Status eines Fiktionalitätssignals und wird zum Hinweis auf die >Gemachtheit< des literarischen Textes, da eine solche, im Moment einer Interviewsituation verankerte Beobachtung als Ergänzung mit mehreren Jahren zeitlicher Distanz unglaubwürdig erscheint.

Deutlicher wird diese Steigerung der Literarizität in dem Kapitel »Barooning «, ${ }^{97}$ das in der überarbeiteten Ausgabe von 2012 neu hinzugekommen ist: Die Anekdote um den Künstlerkollegen David Woodard, ${ }^{98}$ der sich »lautstark eine Dokumentation über japanische Tötungsrituale $\ll{ }^{99}$ anschaut, ${ }^{100}$ wird aus der deklarierten Abwesenheit heraus erzählt. Eingeleitet wird die Anekdote wie folgt: »Als einmal der Komponist David Woodard bei uns zu Gast war, um dem in Kathmandu inhaftierten Charles Sobrajh seine Aufwartung zu machen - wir selbst hielten uns unterdessen im Ausland auf -, hörte Zimmerjunge Krischna einen sehr lauten und langen gellenden Schrei aus der Redaktionsetage. ${ }^{101}$ Die Quelle der folgenden Schilderungen wird nicht benannt und der fiktionale Charakter des Textes wird so narrativ ausgestellt. Auch dieser Steigerung der Fiktionalitätssignale kommt eine gewissermaßen >entstörendeく Funktion zu, da der Text so deutlicher auf den eigenen Status aufmerksam macht und sich gewissermaßen gegen den Vorwurf der Täuschung immunisiert, wenngleich die

\footnotetext{
95 Kracht, Christian/Nickel, Eckhart: Gebrauchsanweisung für Kathmandu und Nepal. [Überarbeitete und erweiterte Neuausgabe] München/Zürich (2012, S. 90).

96 Insbesondere im New Journalism, dem Krachts frühe Arbeiten bei der Zeitschrift Tempo zugerechnet werden, ist dieses an literarischen Formen der Figurencharakterisierung orientierte Stilmittel weit verbreitet: »Die Autoren des New Journalism stehen vor der Herausforderung, real existierende Personen mit den Mitteln literarischer Figurencharakterisierung zu beschreiben. Die Glaubwürdigkeit der Figurengestaltung bildet einen Schlüssel für den Authentizitätscharakter des Textes.« Bleicher, Joan-Kristin: »>Sex, Drugs \& Bücher schreiben $<$. New Journalism im Spannungsfeld von medialem und literarischem Erzählen«. In: Dies./Bernhard Pörksen (Hg.): Grenzgänger. Formen des New Journalism. Wiesbaden (2004, S. 126-159), hier: S. 151.

97 Kracht, Christian/Nickel, Eckhart: Gebrauchsanweisung für Kathmandu und Nepal. [Überarbeitete und erweiterte Neuausgabe] München/Zürich (2012, S. 167-178).

98 Ausschnitte der E-Mail-Korrespondenz von Kracht und David Woodard wurden 2011 unter dem Titel Five Years publiziert: Kracht, Christian/Woodard, David: Five Years. Briefwechsel 2004-2009. Vol. 1: 2004-2007. Hg. von Johannes Birgfeld/Claude D. Conter [Mit einem Vorwort von Johannes Birgfeld und Claude D. Conter], Hannover (2011).

99 Kracht, Christian/Nickel, Eckhart: Gebrauchsanweisung für Kathmandu und Nepal. [Überarbeitete und erweiterte Neuausgabe] München/Zürich (2012, S. 173).

100 Die Beschreibung, in der sich Woodard die Dokumentation anschaut, dazu mit »einer besonderen Tinte« (Kracht, Christian/Nickel, Eckhart: Gebrauchsanweisung für Kathmandu und Nepal. [Überarbeitete und erweiterte Neuausgabe] München/Zürich 2012, S. 173) Notenblätter beschriftet und rote Farbspritzer an der Wand beschrieben werden, erinnert an die gefilmte rituelle Selbsttötung in Die Toten und das »kirschrote Blut«, das die Bildrolle an der Wand bespritzt. Vgl. Kracht, Christian: Die Toten. Roman. Köln (2016, S. 12).

101 Kracht, Christian/Nickel, Eckhart: Gebrauchsanweisung für Kathmandu und Nepal. [Überarbeitete und erweiterte Neuausgabe] München/Zürich (2012, S. 173).
} 
Diskrepanz zwischen dem normalisierten Peritext und dem Haupttext noch verstärkt wird.

Am deutlichsten wird diese Ausstellung der Fiktionalitätssignale im Kapitel »Wege nach Nepal«, das nun um eine längere Passage zu Tony Wheelers Lonely-PlanetReiseführern und deren Auswirkungen ergänzt wurde, die von den Autoren für eine »soziokulturelle Verwüstungsorgie des gesamten Planeten ${ }^{102}$ verantwortlich gemacht werden. Bereits in der Erstausgabe mokieren sich die Autoren über den »moralischen Spagat [...], einen Porter/Sherpa anzuheuern, der recht ergeben das Gepäck auf den Berg hinauf- und hinabträgt, dafür sehr wenig Geld erhält, was eben ein bißchen so aussieht, als sei er, nun ja, ein Sklave. Tony Wheelers dialektisches Genie zeigte sich durch seine Erklärung, die Porter/Sherpas seien eben auf diese Arbeit angewiesen [...]. «103 Dieser Gedanke wird in der Neuausgabe ausgerechnet durch den Verweis auf eine Fälschung ergänzt, die die Autoren auch zu ihrem Text inspiriert haben dürfte:

Was passiert, wenn man nicht nur den Sherpa unterbezahlt, sondern auch die Lohnschreiber, die einen Reiseführer nach dem anderen produzieren müssen, um zu überleben, zeigt das Beispiel Kolumbien. Hier war der zuständige Autor so abgebrannt, daß er, dem alten und schönen Prinzip knowing without going folgend, einfach den gesamten Band aus der Phantasie on a shoestring von zu Hause aus schrieb - ohne je da gewesen zu sein. Man muss überhaupt nicht mehr weit reisen, um World Junk Culture zu bestaunen. ${ }^{104}$

Ein Co-Autor des Lonely-Planet-Reiseführers zu Kolumbien, Thomas Kohnstamm, hatte seinen Beitrag teilweise aus anderen Reiseführern abgeschrieben, teilweise frei erfunden, von zu Hause aus in San Francisco geschrieben und Kolumbien auch noch nie bereist. ${ }^{105}$ Diese Enthüllung, die also bereits ein Jahr vor der Pu-

102 Kracht, Christian/Nickel, Eckhart: Gebrauchsanweisung für Kathmandu und Nepal. München/Zürich (2009, S. 27).

103 Kracht, Christian/Nickel, Eckhart: Gebrauchsanweisung für Kathmandu und Nepal. München/Zürich (2009, S. 26).

${ }^{104}$ Kracht, Christian/Nickel, Eckhart: Gebrauchsanweisung für Kathmandu und Nepal. [Überarbeitete und erweiterte Neuausgabe] München/Zürich (2012, S. 27) [Hervorhebung im Original].

105 Thomas Kohnstamm enthüllte diesen >Fakeくsowie weitere moralisch fragwürdige Praktiken während seiner Tätigkeit für Lonely Planet im Zuge der Publikation seines autobiografischen Textes Do Travel Writers Go to Hell? im April 2008 in einem Interview mit dem Sunday Telegraph selbst: »They didn't pay me enough to go (to) Colombia. I wrote the book in San Francisco. I got the information from a chick I was dating - an intern in the Colombian Consulate. Z Zit. n.: Nizza, Mike: "Lonely Planet Answers a Writer's Claims «. In: The New York Times Online 14.04.(2008), https://thelede.blogs.nytimes.com/2008/ 04/14/lonely-planet-answers-a-writers-claims/ (10.11.2017).

Vgl. reh/Reuters: »Lonely-Planet-Autor gibt Fälschung zu. Viele Urlauber vertrauten seinen Tipps: Ein Autor der [sic] Verlags Lonely Planet gibt an, Inhalte für Reiseführer abgeschrieben und sogar erfunden zu haben. In einem Fall sei er nicht einmal in dem Land gewesen, über das er schrieb - Informationen bekam er demnach von einer Frau, mit der er ausging «. In: Spiegel Online 13.4.(2008), http://www.spiegel.de/ reise/aktuell/kultreisefuehrer-lonely-planet-autor-gibt-faelschungen-zu-a-547054.html (10.11.2017). Siehe auch: Nayhauss, Anette: »Lonely Planet ist plötzlich in Erklärungsnot. Sie sind die >Bibel der Rucksackreisenden $<$, beinahe jedem Backpacker scheinen die >Lonely Planet<-Reiseführer unverzichtbar. Ihre Autoren gelten als unbestechlich, deren Recherchen hautnah und selbsterlebt. Doch jetzt kommt heraus, dass ein Autor abgeschrieben hat - aus anderen Reiseführern«. In: Welt Online 14.4.(2008), https://www. welt.de/reise/article1900647/Lonely-Planet-ist-ploetzlich-in-Erklaerungsnot.html (10.11.2017). 
blikation der Erstausgabe, im April 2008, bekannt wurde, aber als Ergänzung erst Eingang in die Neuausgabe gefunden hat, kann als signifikanter Hinweis auf die Machart des Textes und somit als Kommentar auf die Erstausgabe gelesen werden. Zudem legt die Formulierung dieser Passage eine Übertragung auf die Autoren des $>$ Reiseführers nahe, in dem darauf verwiesen wird: Handelt das reale Beispiel von nur einem Autor, lässt der Plural »die Lohnschreiber« aufhorchen; außerdem habe Kohnstamm, Kracht und Nickels Formulierung zufolge, den ganzen Band frei erfunden, obwohl es sich in dem realen Fall nur um einen Teil des in Co-Autorschaft verfassten Reiseführers handelte. Hier wird also nahegelegt, dass Kracht und Nickel selbst »einfach den gesamten Band aus der Phantasie [...] von zu Hause aus «106 geschrieben haben. »Knowing without going « verweist zudem auf Ferien für immer zurück, das nach Angabe von Kracht und Nickel zunächst diesen Titel hätte tragen sollen: »Da wir ein vergleichsweise geringes Honorar zu erwarten hatten (die Popliteratur war damals noch nicht erfunden), sollte der Arbeitstitel jener Weltbegehung $>$ Knowing without Going lauten und eine Reise im Kopf oder zumindest in der eigenen Wohnung mit eher diffusen Ferienerinnerungen vereinigen. «107

Das Irritationspotential des Textes ergibt sich also vorwiegend daraus, dass der stark literarisierte und teilweise fiktive Inhalt gegen die beglaubigende Form eines Bandes dieser Reihe arbeitet und umgekehrt. Dies ist ein wiederkehrender Aspekt der Reiseliteratur von Kracht und Nickel, nämlich dass die in der Theorie zur Reiseliteratur übliche Grenzziehung zwischen fiktionaler Reiseliteratur, die nicht zwingend auf einer nachweisbar stattgefundene Reise basiert, und nichtfiktionalen Reiseberichten, die auf nachweisbar stattgefundenen Reisen beruhen, ad absurdum geführt wird. ${ }^{108}$ »Knowing without going « meint hier also nicht, dass Kracht und Nickel nicht in Nepal waren, sondern eher, dass dies für die Verfasstheit des Textes kaum relevant zu sein scheint, denn der Text ist »von zu Hause aus « geschrieben und es ist, zugespitzt formuliert, nicht relevant, wo sich dieses zu Hause gerade befindet. ${ }^{109}$

\footnotetext{
106 Kracht, Christian/Nickel, Eckhart: Gebrauchsanweisung für Kathmandu und Nepal. [Überarbeitete und erweiterte Neuausgabe] München/Zürich (2012, S. 27).

107 Kracht, Christian/Nickel, Eckhart: »[Rez. von] Ilija Trojanow: >Der Weltensammler««. In: Der Freund 3 (2006), H. 8, S. 76f., hier: S. 76.

108 Diese Unterscheidung kann in der Reiseliteraturforschung wenn nicht als Konsens, so doch als dominante Position ausgemacht werden und findet sich in einschlägigen Literaturlexika wie dem Reallexikon der deutschen Literaturwissenschaft, dem Metzler Lexikon Literatur und dem Literaturwissenschaftlichen Lexikon. Eine andere Position vertritt bspw. Barbara Korte, die eine »grundsätzliche Fiktionalität « von Reiseliteratur - also auch Reiseberichten - annimmt. Korte, Barbara: Der englische Reisebericht. Von der Pilgerfahrt bis zur Postmoderne. Darmstadt (1996, S. 14). Vgl. Jäger, Hans-Wolf: »Reiseliteratur«. In: JanDirk Müller (Hg.): Reallexikon der deutschen Literaturwissenschaft. Bd. 3. Berlin u.a. (2003, S. 258-261). Vgl. Schuster, Jörg: »Reisebericht«. In: Dieter Burdorf/Christoph Fasbender/Burkhard Moennighoff (Hg.): Metzler Lexikon Literatur. Begriffe und Definitionen. Stuttgart/Weimar ${ }^{3}$ (2007, S. 640f). Vgl. Holdenried, Michaela: »Reiseliteratur«. In: Horst Brunner/Rainer Moritz (Hg.): Literaturwissenschaftliches Lexikon. Grundbegriffe der Germanistik. Berlin 2(2006, S. 226-338). Für einen Forschungsüberblick zur Reiseliteratur siehe: Görbert, Johannes: Die Vertextung der Welt. Forschungsreisen als Literatur bei Georg Forster, Alexander von Humboldt und Adelbert von Chamisso. Berlin (2014, S. 9-18).

109 Bereits ein sehr früher >Reisebericht< Krachts über die Partyszene auf der Insel Sylt erweist sich als eine Art Fake-Reiseführer. Vgl. Kracht, Christian: »Sylt. American \& Dorfkrug«. In: Tempo (1991), H. 6, S. 52 .
} 
Die Neuausgabe weist damit mehrere textuelle wie paratextuelle Strategien und Markierungen auf, die entstörend fungieren. Verlagsseitig scheint eine Korrektur von Fehlern veranlasst worden zu sein, die Falschaussagen im Klappentext korrigiert. Das Erscheinungsbild der Reihe wird zumindest vordergründig wiederhergestellt, was darauf schließen lässt, dass der Verstoß der Autoren gegen bestimmte Konventionen nunmehr auch bei Piper erkannt wurde. Im Haupttext des Buches legt der Verweis auf den gefakten Lonely-Planet-Führer das Verfahren offen, so dass auch nicht literarisch Interessierte spätestens im zweiten Kapitel - wo das »alte[] und schöne[] Prinzip knowing without going «110 beschworen wird - erkennen können, welcher Status dem Text zukommt. Zugleich erweist sich das Störpotential als noch einmal gesteigertes für all jene, die alle Signale überlesen, da nun vor allem die Peritexte weitgehend ihres offenkundigen Unsinns beraubt sind, der als Fiktionalitätsmarker fungierte.

Dies gilt auch für das Cover der Erstausgabe, das höchstwahrscheinlich vom Verlag für die Neuausgabe dem Erscheinungsbild der Reihe entsprechend ausgetauscht wurde. Mit dieser editorischen Maßnahme wurde die Erkennbarkeit, es hier mit Literatur zu tun zu haben, ebenfalls weiter erschwert. Ganz überwiegend zeigen die mittlerweile rund 100 erschienenen Bände der Piper-Reihe Landschaften und Menschen der beschriebenen Länder. Uli Franz' Gebrauchsanweisung für Tibet ist mit dem Bild eines Tibeters in einem farbenfrohen Gewand ein typisches Beispiel hierfür. Die Gebrauchsanweisung für Kathmandu und Nepal ist, soweit wir sehen, die einzige, die ein Schwarzweißfoto ziert. Als Urheber wird ein James Burke ausgewiesen, das Motiv zeige die Nyatapole-Pagode in Bhaktapur. Es erscheint müßig, zu erwähnen, dass dieser Ort - der übrigens auch »Tempel der Schrecklichen « genannt wird - im Buch natürlich gar nicht beschrieben wird. Aufschlussreich ist jedoch der Kontext, in dem das Coverfoto entstand: Der Fotograf James Burke ${ }^{111}$ war Mitte des 20. Jahrhunderts der Indienkorrespondent von LIFE, dem bekanntesten amerikanischen Reportagemagazin, was ihn biografisch mit Christian Kracht verbindet, der Mitte der 90er Jahre Indienkorrespondent des wichtigsten deutschen Nachrichtenmagazins Der Spiegel war. James Burke berichtete etwa 1953 aus Nepal anlässlich der Erstbesteigung des Mount Everest durch Sir Edmund Hillary und den Sherpa Tenzing Norgay.

Burkes Foto auf dem Nepal-Reiseführer entstand im März 1955 und ist in der Datenbank des LIFE Magazine mit folgender Information ausgewiesen: »Tourist in Nepal taking a picture of a temple. «112 Jener namenlose Tourist gehörte zu den frühesten Besuchern, die Nepal nach der Öffnung des Landes bereisten. Es waren Pauschaltouristen, die vom Anbieter Thomas Cook nach Nepal gebracht wurden.

\footnotetext{
110 Kracht, Christian/Nickel, Eckhart: Gebrauchsanweisung für Kathmandu und Nepal. [Überarbeitete und erweiterte Neuausgabe] München/Zürich (2012, S. 27) [Hervorhebung im Original].

111 1915-1964.

112 Burke, James: »Tourist in Nepal taking a picture of a temple.« (1955-03). In: https://www.google. com/culturalinstitute/beta/asset/-/cwHqgyQvOR015w (2.11.2017). Das Bild trägt die »Original ID: TimeLife_image_970547《.
} 
Das LIFE Magazine vom 28. März 1955 brachte eine kurze Fotostrecke über »A Cook's Tour into Katmandu «: ${ }^{113}$

The irrepressible stream of tourism, which has upset many as sanctuary, finally broke into remote Nepal. [...] Nepal allowed Thomas Cook \& Sons to fly in a tourist group, consisting largely of Americans who had come into Bombay on a round-the-world steamship tour. Even in Nepal tourists were tourists: one of them dispensed candy Life Savers to Katmandu beggars. ${ }^{114}$

Alle Bilder der kurzen Reportage zeigen nicht Nepal, sondern allein die Touristen, die einzeln oder in der Gruppe etwas betrachten und Fotos schießen. Nicht allein Burkes Verachtung der Touristen nehmen Kracht und Nickel wieder auf, sondern auch dieses Prinzip, wenn sie nicht etwa >die Fremde< beobachten, sondern durch die impliziten wie expliziten Bezugnahmen auf Texte und Bilder, die >der Westen< sich von Südasien gemacht hat, vielmehr zu Beobachtern zweiter Ordnung werden. Als Prinzip ist dieses Beobachten der Beobachter eine Störung der Gattung Reiseliteratur: knowing without going. Der Vorwurf des Eurozentrismus, der aus Rezensionen spricht, die sich an einem angeblichen >Neokolonialismus< der Dandy-Reisenden stören, zielt an diesem Verfahren vorbei. Es reproduziert mitnichten einen westlicharroganten Blick, sondern stellt gerade die Verhaltensweisen und Blicke westlicher Reisender als hochproblematisch aus. Mit der ursprünglichen Wahl der Titelabbildung der Gebrauchsanweisung für Kathmandu und Nepal wird ein Schlüssel für das Verständnis dieses Verfahrens mitgeliefert: Wer den Text nicht als authentische Reise- und Fremdheitserfahrung, sondern als literarischen Text über die Reisenden und ihre Blicke liest, für den wird auch das Störpotential des Werks sicht- und handhabbar.

Dabei beziehen die Texte Christian Krachts, die bis hin zu seinem Romanwerk (etwa dem umstrittenen Roman Imperium über die historische >Deutsche Südsee $<$ ) sehr häufig nach diesem Muster funktionieren, ${ }^{115}$ einen Gutteil ihres Reizes gerade aus dem Umstand, dass der sich kundig wähnende Leser sich daran freuen kann, er habe eine Spielanordnung durchschaut, der andere Leser - allen voran jene, die sich von Krachts Werken provoziert fühlen - auf den Leim gegangen seien. Tatsächlich ist der Band von Kracht und Nickel eine Invektive gegen die Klientel der PiperReihe, die für ihre eigene Beleidigung sogar noch zur Kasse gebeten wird. Nun wird man aber die Berechtigung literarischer Verfahren nicht daran messen können, dass sie möglicherweise von bestimmten Lesenden nicht (als solche) verstanden

\footnotetext{
113 [Vermutlich James Burke:] »A Cook’s Tour into Katmandu«. In: Life 28.03.1955, S. 43-46, https:// books.google.de/books?id=FlQEAAAAMBAJ\&pg=PA43\&source=gbs_toc_r\&redir_esc=y\#v=onepage \& $\mathrm{q} \& \mathrm{f}=$ false $(2.11 .2017)$.

114 [Vermutlich James Burke:] »A Cook's Tour into Katmandu«. In: Life 28.03.1955, S. 43-46, hier: S. 43, https://books.google.de/books?id=FlQEAAAAMBAJ\&pg=PA43\&source=gbs_toc_r\&redir_esc=y\#v=one page \&q\&f=false (2.11.2017). - Life Savers sind eine amerikanische Bonbonmarke, der Tourist hatte also an Hungernde Süßigkeiten verteilt.

115 Vgl. hierzu etwa Lorenz, Matthias N.: »>Au weia. Kein Frühstück ohne Papaya.< Poetiken des Pazifiks bei Christian Kracht und Hans Christoph Buch«. In: Johannes Görbert/Mario Kumekawa/Thomas Schwarz (Hg.): Pazifikismus. Poetiken des Stillen Ozeans. Würzburg (2017) (= Rezeptionskulturen in Literatur- und Mediengeschichte, Bd. 9), S. 461-479.
} 
werden. Ihre Berechtigung bezieht die auf den ersten Blick so anarchisch-nonsenshafte Gebrauchsanweisung für Kathmandu und Nepal vielmehr aus dem Umstand, dass dieser Band, den die Autoren dem Piper-Verlag offensichtlich erfolgreich untergeschoben haben - wovon die Entstörungsversuche der Neuausgabe zeugen -, die Frage aufwirft, wie es eigentlich sein kann, dass all jene anderen, für die Reihe repräsentativen Bände mit ihren haarsträubenden rassistischen Essentialisierungen und ihrem verqueren eurozentrischen Orientalismus ohne jede Skandalisierung erscheinen konnten. Vor diesem Hintergrund wäre der Band als eine kritische, durchaus politische Intervention zu lesen, die in gewisser Weise analog zu der Social-MediaKampagne »Hass hilft« verläuft, bei der Posts mit rassistischen Invektiven gegen Geflüchtete automatisch Spenden zu deren Gunsten auslösen. Ähnlich überlistet die Gebrauchsanweisung für Kathmandu und Nepal Käufer der Piper-Reihe, die, nachdem sie 14,99 Euro ausgegeben haben, mit den für diese Reihe und ihre Klientel offenbar typischen Projektionen konfrontiert werden, anstatt im Fernweh schwelgen zu dürfen. Diese Ähnlichkeit findet aber ihre Grenze in dem nicht unerheblichen Umstand, dass die Aktivistinnen und Aktivisten von »Hass hilft« ihre Kampagne transparent führen, ihr Engagement offenlegen und die eingenommenen Gelder wie erwähnt spenden. Dagegen adressieren Kracht und Nickel, die eher spielerisch eine Art Wahrnehmungsfalle konstruiert haben, keineswegs gezielt Rassistinnen und Rassisten, sondern schließen in letzter Konsequenz umstandslos von einer Reihe, in der einige sehr problematische Bände erschienen sind, auf deren Leserinnen und Leser insgesamt. Allen jedoch, deren Rezeptionserwartungen so getäuscht und enttäuscht wurden, bieten die Autoren gleichwohl eine im besten Falle vergnügliche Lektüre. ${ }^{116}$

\section{Literatur}

[Vermutlich James Burke:] »A Cook's Tour into Katmandu«. In: Life 28.3.1955, S. 43-46, https://books. google.de/books?id=FlQEAAAAMBAJ\&pg=PA43\&source=gbs_toc_r\&redir_esc=y\#v=onepage\& $\mathrm{q} \& \mathrm{f}=$ false $(2.11 .2017)$.

Amend, Christoph/Lebert, Stephan: »Ich weine oft«. In: Der Tagesspiegel 2.7.2000, S. W1 [Weltspiegel]. Birgfeld, Johannes/Conter, Claude D. (Hg.): Christian Kracht. Zu Leben und Werk. Köln 2009.

Bleicher, Joan-Kristin: »)Sex, Drugs \& Bücher schreiben<. New Journalism im Spannungsfeld von medialem und literarischem Erzählen«. In: Dies./Bernhard Pörksen (Hg.): Grenzgänger. Formen des New Journalism. Wiesbaden 2004, S. 126-159.

Boeck, Julia: »Die Freunde. Eine Gebrauchsanweisung für Kathmandu und Nepal«. In: Der Tagesspiegel 3.5.2009, S. 31.

\footnotetext{
116 Einzelne Kundenrezensentinnen oder -rezensenten konnten sich auf diese einlassen und gaben auf amazon.de die bestmögliche Bewertung für den Text ab; sie rezipierten den Text explizit nicht als Reiseführer: »Kann überhaupt nicht nachvollziehen, weshalb das Buch so schlecht bewertet wird. Wer einen Reiseführer erwartet, wird enttäuscht. Wer ohne Erwartungen liest, wird überrascht.« (Rezension von »jaegermeister« auf amazon.de, 24.6.2015. In: https://www.amazon.de/Gebrauchsanweisung-f\%C3\%BCr-KathmanduNepal-\%C3\%9Cberarbeitete/product-reviews/3492276156/ref=cm_cr_dp_d_show_all_btm?ie=UTF8\&. reviewerType=all_reviews (29.5.2017)). In einem weiteren Beispiel wird der Text uneingeschränkt und selbst ironisch als »Juwel der Reiseliteratur« empfohlen (Rezension von »mvonotto« auf amazon.de, 18.8.2011. In: https://www.amazon.de/Gebrauchsanweisung-Kathmandu-Nepal-Christian-Kracht/dp/ 3492275648/ref=sr_1_2?ie=UTF8\&qid=1496051686\&sr=8-2\&keywords=gebrauchsanweisung $+\mathrm{f} \% \mathrm{C} 3$ $\% \mathrm{BCr}+\mathrm{kathmandu+und+nepal} \mathrm{(29.5.2017)).}$
} 
Burke, James: »Tourist in Nepal taking a picture of a temple.« (1955-03). In: https://www.google.com/ culturalinstitute/beta/asset/-/cwHqgyQvOR015w (2.11.2017).

Douglas, Mary: Purity and Danger. An Analysis of Concepts of Pollution and Taboo. New York 1966.

Facebook-Seite von Der Freund. In: https://www.facebook.com/der.freund.magazine/photos/a.10151583 442527445.1073741826.54088502444/10153692584962445/?type=3\&theater (12.11.2017).

Franz, Uli: Gebrauchsanweisung für Tibet. [überarbeitete Neuausgabe] München/Zürich 2007.

Gansel, Carsten: »Einleitung«. In: Ders./Norman Ächtler (Hg.): Das »Prinzip Störung« in den Geistesund Sozialwissenschaften. Berlin 2013, S. 7-13.

Görbert, Johannes: Die Vertextung der Welt. Forschungsreisen als Literatur bei Georg Forster, Alexander von Humboldt und Adelbert von Chamisso. Berlin 2014.

Hachhethu, Krishna/Gellner, David N.: »Nepal. Trajectories of democracy and restructuring of the state«. In: Paul R. Brass (Hg.): Routledge Handbook of South Asian Politics. India, Pakistan, Bangladesh, Sri Lanka, and Nepal. London/New York 2010, S. 131-146.

Harrer, Heinrich: Sieben Jahre in Tibet. Mein Leben am Hofe des Dalai Lama. Wien 1952.

Hergé: Tim in Tibet (= Tim und Struppi, Bd. 19). Hamburg 1999.

Hesse, Hermann: »Die Morgenlandfahrt. Eine Erzählung«. In: Ders.: Gesammelte Dichtungen. Sechster Band. Frankfurt am Main/Zürich 1952.

Holdenried, Michaela: »Reiseliteratur«. In: Horst Brunner/Rainer Moritz (Hg.): Literaturwissenschaftliches Lexikon. Grundbegriffe der Germanistik. Berlin ${ }^{2} 2006$, S. 226-338.

Jäger, Hans-Wolf: »Reiseliteratur«. In: Jan-Dirk Müller (Hg.): Reallexikon der deutschen Literaturwissenschaft. Bd. 3. Berlin u.a. 2003, S. 258-261.

Jünger, Ernst: Das zweite Pariser Tagebuch. In: Ders.: Sämtliche Werke. Tagebücher III. Strahlungen II. Stuttgart 1979, S. 9-294.

Kiesel, Helmuth: Ernst Jünger. Die Biographie. München 2007.

Korte, Barbara: Der englische Reisebericht. Von der Pilgerfahrt bis zur Postmoderne. Darmstadt 1996.

Kracht, Christian: »Sylt. American \& Dorfkrug«. In: Tempo (1991), H. 6, S. 52.

Kracht, Christian/Nickel, Eckhart: »[Rez. von] Ilija Trojanow: >Der Weltensammler««. In: Der Freund 3 (2006), H. 8, S. $76 f$.

Kracht, Christian/Nickel, Eckhart: Ferien für immer. Die angenehmsten Orte der Welt. [Mit einem Vorwort von Moritz von Uslar] Köln 1998.

Kracht, Christian/Nickel, Eckhart: Gebrauchsanweisung für Kathmandu und Nepal. [Überarbeitete und erweiterte Neuausgabe] München/Zürich 2012.

Kracht, Christian/Nickel, Eckhart: Gebrauchsanweisung für Kathmandu und Nepal. München/Zürich 2009.

Kracht, Christian/Woodard, David: Five Years. Briefwechsel 2004-2009. Vol. 1: 2004-2007. Hg. von Johannes Birgfeld/Claude D. Conter [Mit einem Vorwort von Johannes Birgfeld und Claude D. Conter]. Hannover 2011.

Kracht, Christian: Die Toten. Roman. Köln 2016.

Lorenz, Matthias N.: »Der freundliche Kannibale. Über den Provokationsgehalt der Figur >Christian Kracht««. In: Merkur. Deutsche Zeitschrift für europäisches Denken 68 (2014), H. 11, S. 1022-1026.

Lorenz, Matthias N.: » Au weia. Kein Frühstück ohne Papaya.< Poetiken des Pazifiks bei Christian Kracht und Hans Christoph Buch«. In: Johannes Görbert/Mario Kumekawa/Thomas Schwarz (Hg.): Pazifikismus. Poetiken des Stillen Ozeans. Würzburg 2017 (= Rezeptionskulturen in Literatur- und Mediengeschichte, Bd. 8), S. 461-479.

Lütkehaus, Ludger: »Grenzüberschreitung«. In: Neue Zürcher Zeitung 1.7.2000, S. 68.

Malchow, Helge/Kleinschmidt, Christoph: »Hermeneutik des Bruchs oder Die Neuerfindung frühromantischer Poetik. Ein Gespräch«. In: Christoph Kleinschmidt (Hg.): Text+Kritik. Zeitschrift für Literatur. Christian Kracht (2017), H. 216, S. 34-43.

Müller, Burkhard: »Geklauter Portwein gibt eine tolle Geschichte. Ausgewählt nach privatem Schrägheitsindex: Christian Kracht und Eckhart Nickel berichten von ihrer Zeit in Nepal«. In: Süddeutsche Zeitung 27.4.2009, S. 14.

Nayhauss, Anette: »Lonely Planet ist plötzlich in Erklärungsnot«. In: Welt Online, 14.04.2008, https:// www.welt.de/reise/article1900647/Lonely-Planet-ist-ploetzlich-in-Erklaerungsnot.html (10.11.2017).

Nickel, Eckhart: Gebrauchsanweisung für Portugal. München/Zürich ${ }^{5} 2010$.

Nizza, Mike: »Lonely Planet Answers a Writer's Claims«. In: The New York Times Online, 14.04.2008, https://thelede.blogs.nytimes.com/2008/04/14/lonely-planet-answers-a-writers-claims/ (10.11.2017).

reh/Reuters: »Lonely-Planet-Autor gibt Fälschung zu«. In: Spiegel Online, 13.04.2008, http://www. spiegel.de/reise/aktuell/kultreisefuehrer-lonely-planet-autor-gibt-faelschungen-zu-a-547054.html (10.11.2017). 
Rottmann, Markus: »Ein Shanti von oben herab«. In: Schweizer Monat 6 (2012), H. 7, o.S. In: https:// www.schweizermonat.ch/artikel/ein-shanti-von-oben-herab (12.11.2017).

Schuster, Jörg: »Reisebericht«. In: Dieter Burdorf/Christoph Fasbender/Burkhard Moennighoff (Hg.): Metzler Lexikon Literatur. Begriffe und Definitionen. Stuttgart/Weimar ${ }^{3} 2007$, S. 640f.

Shrestha, Nanda R./Bhattararai, Keshav: Historical Dictionary of Nepal. Lanham u.a. ${ }^{2} 2017$ (= Historical Dictionaries of Asia, Oceania, and the Middle East).

Strittmatter, Kai: Gebrauchsanweisung für China. München/Zürich ${ }^{3} 2010$.

Trojanow, Ilija: Gebrauchsanweisung für Indien. München/Zürich ${ }^{3} 2007$.

Website von amazon.de., Gebrauchsanweisung für China (2008). In: http://www.amazon.de/Gebrauchs anweisung-für-China-Kai-Strittmatter/dp/3492275745/ref=sr_1_1 ?ie=UTF8\&qid=1512493460\&sr= 8-1\&keywords=gebrauchsanweisung+für+china (5.12.2017).

Website von amazon.de., Gebrauchsanweisung für Kathmandu und Nepal (2009). In: https://www. amazon.de/Gebrauchsanweisung-Kathmandu-Nepal-Christian-Kracht/dp/3492275648/ref=sr_1_2? ie $=\mathrm{UTF} 8 \&$ qid $=1496051686 \& \mathrm{sr}=8-2 \&$ keywords $=$ gebrauchsanweisung $+\mathrm{f} \% \mathrm{C} 3 \% \mathrm{BCr}+\mathrm{kathmandu}+$ und+nepal (29.5.2017).

Website von amazon.de., Gebrauchsanweisung für Kathmandu und Nepal (2012). In: https://www.amazon. de/Gebrauchsanweisung-f\%C3\%BCr-Kathmandu-Nepal-\%C3\%9Cberarbeitete/product-reviews/34 92276156/ref=cm_cr_arp_d_paging_btm_2?ie=UTF8\&reviewerType=all_reviews\&pageNumber=2 (29.05.2017)

Website von Der Freund, Rubrik »Presse«. In: http://www.derfreund.com/presse.php (13.11.2017).

Website des Piper-Verlags. In: https://www.piper.de/buecher/abenteuer-reiseberichte/gebrauchsanweisung (2.11.2017).

Website des Projekts »Poets translating Poets. Poetry traverses South Asia and Germany«. In: http://www. goethe.de/ins/in/lp/prj/ptp/tra/en15346004.htm (12.11.2017).

Whelpton, John: A History of Nepal. Cambridge 2005.

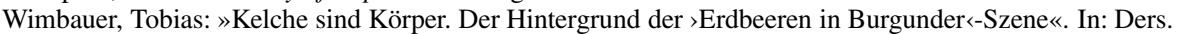
(Hg.): Anarch im Widerspruch. Neue Beiträge zum Werk und Leben der Gebrüder Jünger. Schnellroda 2004, S. 23-69.

Winkels, Hubert (Hg.): Christian Kracht trifft Wilhelm Raabe. Die Diskussion um »Imperium« und der Wilhelm Raabe-Literaturpreis 2012. Berlin 2013. 